\title{
The Enigmatic HCl + Au(111) Reaction: A Puzzle for Theory and Experiment
}

\author{
Gernot Füchsel $^{1 *}$, Marcos del Cueto $^{2}$, Cristina Díaz ${ }^{2,3}$, and Geert-Jan Kroes ${ }^{1 * *}$
}

1 Leiden Institute of Chemistry, Gorlaeus Laboratories, Leiden University, P.O. Box 9502, 2300 RA Leiden, The Netherlands

2 Departamento de Química, Módulo 13, Universidad Autónoma de Madrid, 28049

Madrid, Spain

3 Condensed Matter Physics Center (IFIMAC), Universidad Autónoma de Madrid, 28049 Madrid, Spain

\author{
Corresponding authors: \\ *g.fuchsel@chem.leidenuniv.nl, Tel.: +31715274533 \\ **g.j.kroes@chem.leidenuniv.nl, Tel.: +31715274396
}

\begin{abstract}
.
The reactive scattering of $\mathrm{HCl}$ on $\mathrm{Au}(111)$ is currently one of the most peculiar reactions in the field of surface chemistry, as it so far eludes an accurate theoretical description. Possible reasons for the observed mismatch between theory and experiment that were not yet all considered in the computations are i) dissipative effects due to the creation of electron hole pairs and excitations of surface atom motion that might inhibit reaction, and ii) the use of an inappropriate density functional theory method, or even its failure due to the occurrence of a charge transfer at the transition state. In this work, we address all of these possibilities and perform quasiclassical molecular dynamics simulations employing different methodologies. We use ab initio molecular dynamics simulations to account for surface atom motion and surface temperature effects, employing the PBE and the RPBE functionals. We also construct
\end{abstract}


an accurate potential energy surface incorporating the six adsorbate degrees of freedom of $\mathrm{HCl}$ on $\mathrm{Au}(111)$ by using the neural network approach. In our molecular dynamics simulations, we model the experimental beam conditions to study the influence of the rovibrational state population distribution of $\mathrm{HCl}$ in the molecular beam on reaction. Likewise, molecular dynamics with electronic friction calculations based on the parameterfree local density friction approximation in the independent atom approximation (LDFA-IAA) are performed to get first insights into how electron hole pair excitation might affect the reactivity of $\mathrm{HCl}$. Although satisfying agreement with the experiment could not yet be achieved by our simulations, we find that i) RPBE yields larger reaction barriers than PBE and lower computed reaction probabilities, improving the agreement with experiment, ii) the reactivity strongly depends on the rovibrational state population, iii) surface atom motion and electronically non-adiabatic effects modeled with the efficient to use but approximate LDFA influence the reaction only modestly, and iv) a moderate amount of charge is transferred from the surface to the dissociating molecule at the transition state. We suggest that the reported experimental reaction probabilities could be too low by a factor of about 2-3, due to potential problems with calibrating coverage of $\mathrm{Au}$ by $\mathrm{Cl}$ using an ill-defined external standard in the form of Auger peak ratios. However, taking this factor into account would not yet resolve the discrepancy between the theoretical reaction probabilities presented here and the experimental sticking probabilities.

\section{Introduction}

Theoretical work has shown that, in a system in which an atom or molecule moves towards a metal surface, kinetic energy in the nuclear motion must be dissipated to electron-hole pair (ehp) excitations ${ }^{1}$. However, this does not necessarily imply that this electronically non- 
adiabatic effect has a large impact on the scattering dynamics, and that electronically adiabatic theory should be largely inaccurate for the dynamics in such systems ${ }^{2}$. Nonetheless considerable evidence has been gathered from both experimental and theoretical studies that many such systems are considerably affected by electronically non-adiabatic effects. Examples include chemicurrents measured when atoms and molecules are scattered from a polycrystalline silver surface ${ }^{3}$, multi-quantum vibrational relaxation of highly vibrationally excited NO scattering from $\mathrm{Au}(111)^{4,5}$, vibrationally promoted electron emission ${ }^{6,7}$, and $\mathrm{H}-$ atoms scattering from and sticking on $\mathrm{Au}(111)^{8-10}$.

Persuasive evidence that dissociative chemisorption of molecules on metal surfaces should be strongly affected by electronically non-adiabatic effects has, however, been missing. For some reactions, like $\mathrm{H}_{2}+\mathrm{Cu}(111)^{11}, \mathrm{H}_{2}+\mathrm{Cu}(100)^{12}, \mathrm{H}_{2}+\mathrm{Ru}(0001)^{13}$, and $\mathrm{CHD}_{3}+\mathrm{Ni}(111)^{14}$ it has been shown that dynamics calculations can reproduce molecular beam sticking experiments with chemical accuracy (to within $1 \mathrm{kcal} / \mathrm{mol}$ ) on the basis of density functional theory using semi-empirically determined functionals at the generalized gradient approximation (GGA) level, or GGA inspired functionals. By this, we mean that the computed reaction probability curve is shifted from the experimental sticking curve along the incidence energy axis by no more than $1 \mathrm{kcal} / \mathrm{mol}^{11}$. For other, often more complex reactions, such as $\mathrm{D}_{2} \mathrm{O}+\mathrm{Ni}(111)^{15}$ and methane $+\mathrm{Pt}(111)^{16,17}$, molecular beam sticking experiments are described at least semi-quantitatively by dynamics calculations based on DFT at the GGA level. Also, dynamics calculations using a GGA/DFT potential energy surface (PES) and molecular dynamics with electronic friction (MDEF) generally only find small effects of ehp excitation on dissociative chemisorption reactions ${ }^{18-22}$. It has been argued that the reaction of $\mathrm{O}_{2}$ with $\mathrm{Al}(111)$ should be affected by non-adiabatic effects related to its electronic $\operatorname{spin}^{23,24}$. However, a rival explanation for this systems exists which attributes the failure of GGA/DFT 
to accurately describe $\mathrm{O}_{2}+\mathrm{Al}(111)$ to its predicting too low energies for systems exhibiting electron transfer ${ }^{25}$. More generally, such a problem might be expected to occur if a molecule with a positive electron affinity (like $\mathrm{O}_{2}$ ) collides with a metal with a low work function.

The ability of GGA/DFT to describe dissociative chemisorption on metals with at least semiquantitative accuracy for a range of systems is good news for scientists aiming to describe heterogeneous catalysis from first principles, as the rate limiting step in reactions catalyzed by metals is often a dissociative chemisorption reaction ${ }^{26,27}$. At the same time, it is of clear fundamental interest to identify reactions that might be strongly affected by electronically non-adiabatic effects, and to see if these reactions may also be modeled with good accuracy if suitably accurate methods are selected ${ }^{22}$. Recently, the dissociative chemisorption of $\mathrm{HCl}$ on $\mathrm{Au}(111)$ has been put forward as a potential candidate system ${ }^{28,29}$.

The $\mathrm{HCl}+\mathrm{Au}(111)$ system has been the subject of a large number of experimental and theoretical studies. It is implicated as the product of the Eley-Rideal reaction of $\mathrm{H}$ with $\mathrm{Cl}$ chemisorbed on $\mathrm{Au}(111)$, which has been studied both experimentally ${ }^{30,31}$ and theoretically ${ }^{32-}$ ${ }^{34}$. The rotationally inelastic scattering of $\mathrm{HCl}$ from $\mathrm{Au}(111)$ has also been studied, using both conventional molecular beam techniques ${ }^{35}$ and molecular beams combined with final stateresolved velocity map imaging techniques ${ }^{36}$. Evidence of electronically non-adiabatic effects $^{37-39}$ has been emerging from recent studies of vibrationally inelastic scattering of $\mathrm{HCl}$ from $\mathrm{Au}(111)^{37-40}$. For instance, as a function of surface temperature $\left(T_{s}\right)$ probabilities for vibrational excitation from $v=0$ to $v=1$ show a change in slope at $T_{S}=800 \mathrm{~K}$, which is consistent with a change from an electronically adiabatic to a non-adiabatic mechanism ${ }^{37}$. Molecules scattering from $v=2$ to $v=1$ transfer about $75 \%$ of the vibrational energy released to the surface, which should, in large part, be due to ehp excitation ${ }^{38}$. 
The dissociative chemisorption of $\mathrm{HCl}$ on $\mathrm{Au}(111)$ was first studied theoretically by Zhang and co-workers ${ }^{41-43}$. They used DFT with the PW91 functional ${ }^{44}$ and the neural network method to construct a six-dimensional PES for $\mathrm{HCl}$ interacting with a static $\mathrm{Au}(111)$ surface $^{43}$. In the minimum energy path they determined, at $r \approx 1.92 \AA$ the $\mathrm{HCl}$ bond is considerably extended at the barrier geometry ${ }^{43}$, compared to $r=1.27 \AA$ in the gas phase ${ }^{45}$. In the tilted minimum barrier geometry, $\mathrm{HCl}$ is close to the $f c c$ hollow site with the molecular axis making a polar angle $\theta \approx 48^{\circ}$ with the surface normal, with the H-atom pointing down. With the functional and DFT input parameter set used, the minimum barrier height for dissociation was found to be approximately $0.65 \mathrm{eV}^{43}$. Liu et al. also performed 6D quantum dynamical wave packet calculations of the activated reaction of $(v, j) \mathrm{HCl}^{41,43}$ ( $j$ is the rotational quantum number of $\mathrm{HCl})$. The reaction is promoted by vibrational pre-excitation of the molecule to $v=1$, and pre-alignment of the rotational angular momentum was found to have a pronounced effect on the reactivity, with "helicoptering" $\left(j=5, m_{j}=5\right) \mathrm{HCl}$ being substantially more reactive at high incidence energies $E_{i}$ than "cartwheeling" $\left(j=5, m_{j}=0\right)$ $\mathrm{HCl}^{41}$. Zhang and co-workers also studied the scattering of $\mathrm{DCl}$ from $\mathrm{Au}(111)^{42}$, obtaining similar results as found for $\mathrm{HCl}+\mathrm{Au}(111)^{41}$.

Very recently, the dissociative chemisorption of $\mathrm{HCl}$ on $\mathrm{Au}(111)$ was studied experimentally, using molecular beam techniques ${ }^{29}$. A striking result is that the reaction probability is only about $2 \%$ for $E_{i} \approx 2.5 \mathrm{eV}^{29}$, even though the DFT PW91 calculations put the barrier height at only $0.65 \mathrm{eV}$. Experiments in which $E_{i}$ and the nozzle temperature $T_{n}$ were varied independently suggest that the reaction is promoted by vibrational excitation to such a high extent that the reactivity of $\mathrm{HCl}$ is dominated by $v=1$ reaction over the entire range of $E_{i}$ studied (up to about $2.5 \mathrm{eV})^{29}$. At the value of $T_{s}$ for which the experiments were performed 
(170K), $\mathrm{HCl}$ and $\mathrm{H}_{2}$ recombinative desorption introduce some uncertainty in the measurements of the sticking probability, which were based on determining the coverage of $\mathrm{Au}$ by the product $\mathrm{Cl}^{29}$. However, the authors estimated the uncertainty in the sticking probability to be no more than a factor two, and concluded that the discrepancies between their results ${ }^{29}$ and the PW91 based theoretical results ${ }^{41}$ were so large that deficiencies in the computational approach should be addressed ${ }^{29}$. The uncertainties in the experimental results will be discussed further below.

Possible deficiencies in the theoretical approach taken were considered by both Shirhatti et $a l^{29}$ and by Wodtke ${ }^{28}$. As noted by these authors, first of all, it is possible that the barrier height was underestimated with the PW91 functional ${ }^{44}$ used, which tends to overestimate the reactivity for many molecule + metal systems ${ }^{11-13,16}$. This problem could be compounded if substantial electron transfer takes place from $\mathrm{Au}(111)$ to the extended $\mathrm{HCl}$ molecule near the minimum barrier geometry of the system ${ }^{25,28}$. Secondly, it is possible that on the way to the barrier $\mathrm{HCl}$ loses a lot of its energy in motion towards the surface (which at least initially corresponds to the energy in the reaction coordinate) to the phonons of $\mathrm{Au}(111)$. The application of a binary collision model suggests that about $50 \%$ of this energy could be transferred from the incident $\mathrm{HCl}$ to a surface $\mathrm{Au}$ atom ${ }^{28}$. Thirdly, it is possible that the incident $\mathrm{HCl}$ transfers part of its incidence energy to ehp excitation, the possibility of which process is strongly suggested by the finding of electronically non-adiabatic effects in experiments on vibrationally inelastic scattering of $\mathrm{HCl}$ from $\mathrm{Au}(111)^{37-39}$. As noted above, it is this possibility that conveys a special interest to the $\mathrm{HCl}+\mathrm{Au}(111)$ system, as a dissociative chemisorption reaction that is potentially affected by large electronically nonadiabatic effects. 
Here, we perform a theoretical study of the $\mathrm{HCl}$ on $\mathrm{Au}(111)$ system to address all of these possible effects. We perform $A b$ Initio Molecular Dynamics (AIMD) studies of the dissociative chemisorption reaction. By using the PBE functional ${ }^{46}$ (which yields electronic energies much like $\mathrm{PW} 91^{46}$ ) in some and the more repulsive RPBE functional ${ }^{47}$ in other AIMD calculations, we investigate the effect of the height and, to some extent, the geometry of the minimum barrier on the reaction. We also perform a Bader charge analysis ${ }^{48-51}$ for the minimum barrier geometry to assess whether more charge transfer occurs in the $\mathrm{HCl}+$ $\mathrm{Au}(111)$ minimum barrier geometry than in the barrier geometries of systems like $\mathrm{H}_{2}+$ $\mathrm{Cu}(111)$ and $\mathrm{CH}_{4}+\mathrm{Ni}(111)$. Because in AIMD the surface atoms can be treated as mobile and made to move according to a specific value of $T_{s}$ and to respond to the incident $\mathrm{HCl}$ molecule, the AIMD calculations also allow us to investigate the effect of energy transfer from incident $\mathrm{HCl}$ to the surface phonons. We also use the neural network $\operatorname{method}^{52,53}$ and PBE-DFT to fit a PES and use this in MDEF calculations ${ }^{20}$ to assess the possible effects of ehp excitation. In these calculations, ehp excitation is modeled with electronic friction coefficients computed in the independent atom approximation (IAA) using the local density friction approximation (LDFA) $)^{19}$ - an efficient to use but approximate method which is further discussed in Sec. 2.4. We investigate the effect of the incident translational energy and vibrational state of the molecule on the reaction (also indirectly, through $T_{n}$ ), and the effect of $T_{s}$. We also looked at whether there might be possible effects of the incident rotational state, which were not yet considered in earlier analyses ${ }^{28,29}$. As noted above, the $\mathrm{HCl}+\mathrm{Au}(111)$ reaction has a late barrier geometry, and experiments on $\mathrm{H}_{2}+\mathrm{Cu}(111)$ have suggested that initial rotation of the dissociating molecule might then inhibit dissociation ${ }^{54,55}$. Such an inhibitive effect may arise if the molecule rotates out of its favorable reactive orientation while attempting to traverse the bottleneck presented by the transition state (TS) ${ }^{54,55}$. 
This paper is organized as follows: in Section 2, we present the models and the methodology including AIMD, MD and MDEF simulations, the friction model and the neural network approach. In Section 3, we report on specific properties of the PES for $\mathrm{HCl}$ on $\mathrm{Au}(111)$ and on our main results taken from the dynamics simulations. We also discuss the present level of agreement between theory and experiment, and possible improvements in both. Finally, a summary and a conclusion is given in Section 4.

\section{Methods and models}

We study the dissociative adsorption of $\mathrm{HCl}$ on $\mathrm{Au}(111)$ at normal incidence by performing molecular dynamics simulations without and with electronic friction (MD and MDEF) on a six-dimensional (6D) PES, and, in addition to this, with the help of ab initio molecular dynamics simulations (AIMD). MD simulations performed on a pre-calculated PES are useful to evaluate physical observables, such as reaction probabilities, at a high level of statistical accuracy, but at low computational costs. This is particularly important for calculations on an event that is unlikely to happen. Highly accurate potential energy surfaces, however, are not easily created for complex systems, and are often limited to a certain amount

of dynamical degrees of freedom $(N=6-15)^{56-60}$. An advantage of AIMD simulations is that they circumvent this limitation by calculating the potential energy and the resulting forces on the fly, that is, at each time step of the MD dynamics. This technique makes it possible to incorporate a large number of degrees of freedom in dynamical simulations, but this comes at the cost of a much larger computational burden. Its application is therefore focused on the determination of events that occur with probabilities $>0.01$.

Our dynamics simulations are all performed on the level of classical mechanics with quasiclassical initial conditions, called QCT in the following, to take into account the quantum 
mechanical eigenenergies of the different rovibrational states of $\mathrm{HCl}$ in the gas phase. The use of a classical treatment instead of a quantum treatment is motivated by i) the success of quasiclassical simulations in describing accurately the chemistry of other systems showing similar features, such as the dissociative adsorption of $\mathrm{D}_{2}$ on $\mathrm{Cu}(111)^{61}$ ii) the low computational costs, and iii) the comparably simple incorporation of dissipative effects (phonon and electronic excitation). The reduced masses of $\mathrm{D}_{2}$ and $\mathrm{HCl}$ are very similar $(\approx 1$ $\mathrm{amu}$ ), and so are the vibrational frequencies (the vibrational quanta are $370 \mathrm{meV}$ for $\mathrm{D}_{2}$ and $350 \mathrm{meV}$ for $\mathrm{HCl})$. Also, the $\mathrm{HCl}$ on $\mathrm{Au}(111)$ and the $\mathrm{D}_{2}$ on $\mathrm{Cu}(111)$ systems are both late barrier systems. The fact that the QCT method is accurate for $\mathrm{D}_{2}+\mathrm{Cu}(111)$ then suggests that the method should also be accurate for $\mathrm{HCl}+\mathrm{Au}(111)$.

\subsection{Computational setup}

The present study is carried out by using the Vienna Ab Initio Simulation Package (VASP), version 5.3.5 $5^{62-65}$, which allows for electronic structure calculations within the BornOppenheimer approximation by employing periodic density functional theory (DFT). Two different gradient corrected (GGA) DFT functionals, namely the Perdew-Burke-Ernzerhof $(\mathrm{PBE})^{46}$ and its revised version, the RPBE functional ${ }^{47}$, are used to describe the dissociative adsorption of $\mathrm{HCl}$ on $\mathrm{Au}(111)$. The $\mathrm{Au}(111)$ surface is represented by a (2×2) supercell and a four layer slab model with a vacuum gap of $16 \AA$ between periodically repeated slabs. The ion cores are described with the plane augmented wave (PAW) method ${ }^{66,67}$, and a kinetic energy cut-off of $400 \mathrm{eV}$ is used. We make use of a $11 \times 11 \times 1 \mathrm{k}$-point mesh on a $\Gamma$-centered Monkhorst-Pack grid.

The optimization of the bulk lattice constant yields $L_{B u l k}=4.1554 \AA$ for the PBE functional, and $L_{B u l k}=4.1956 \AA$ for the RPBE functional, both in reasonable agreement with the 
experimental value of $4.0783 \AA^{68}$. The corresponding shortest Au-Au distances are $D=$ $2.9384 \AA$ (PBE) and $D=2.9667 \AA$ (RPBE). Before using the four-layer slab to model the reactive scattering of $\mathrm{HCl}$ on $\mathrm{Au}(111)$, we optimized the interlayer distances $d$ while keeping the lowest $\left(4^{\text {th }}\right.$ ) layer fixed. With the PBE functional, the ideal value of $d$ is $2.399 \AA$. After the surface relaxation, we obtain $d_{12}=2.410 \AA, d_{23}=2.377 \AA$ and $d_{34}=2.411 \AA$. We also find that the classical binding energy $E_{0}$ of $\mathrm{HCl}$ is well described by the employed GGA functionals. At a molecule-surface distance $Z=5.3 \AA$ and for $\mathrm{HCl}$ parallel to the surface, we find that $E_{0}=4.426 \mathrm{eV}$ with $\mathrm{PBE}$, at the $\mathrm{H}-\mathrm{Cl}$ equilibrium distance $r_{e q}=1.282 \AA$, whereas RPBE yields $E_{0}=4.509 \mathrm{eV}$ at the same $r_{e q}$ distance. These values are in good agreement with the experimental binding energy, including zero-point energy ${ }^{69}$, of $E_{0}=4.618 \mathrm{eV}$ at $r_{e q}=1.275 \AA^{70,71}$.

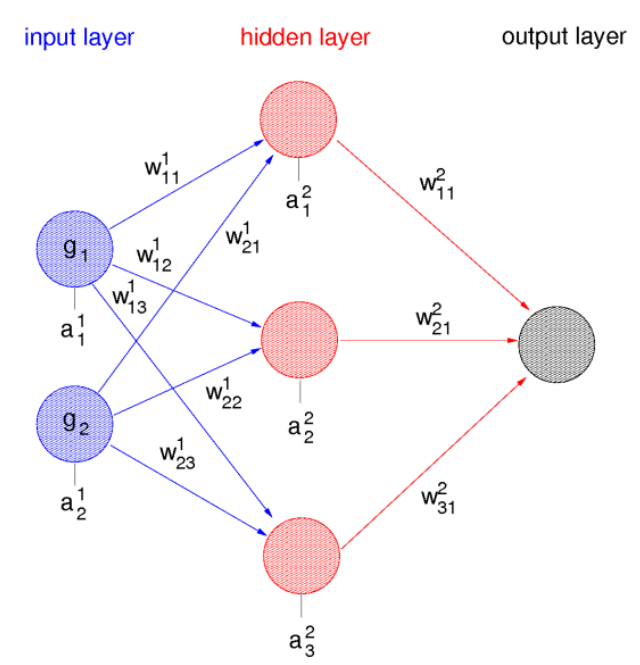

Figure 1: Shown is the topology of a 2-3-1 feedforward neural network consisting of one input layer, one hidden and one output layer. The nodes are interconnected via weights $w_{i j}^{l}$ and the activation functions, the latter being biased according to the bias values $\boldsymbol{a}_{i}^{l}$. 


\subsection{Potential energy surface and coordinate system}

For precise MD and MDEF simulations a continuous representation of the potential energy surface (PES) is needed that allows for an accurate and ideally fast evaluation of the potential energy and the forces. Several methods have been put forward for finding a functional representation of a set of discrete potential values which is able to predict the unknown potential at other points within the precision of the underlying electronic structure theory used in the calculations. An example is an interpolation method based on the corrugation reducing procedure $^{56}$, which is typically used for systems involving diatomic molecules and a surface $^{57,58}$, but also fitting procedures such as force field methods ${ }^{59}$ and the artificial feedforward neural network (NN) approach ${ }^{60,72}$. Although not yet as commonly used to represent PESs, the NN method is considered as a promising technique to accurately fit highdimensional PESs for systems with a very large number of DOF due to its flexibility, universality and learning capabilities. Before describing in detail how we apply this method in order to obtain a 6D PES for $\mathrm{HCl}$ interacting with a rigid $\mathrm{Au}(111)$ surface, we first briefly describe its main principles.

Although modifications inspired by the physics of the problem can be made, the use of NNs does not necessitate any assumptions about the physics dictating the shape of the function to be fitted. Feedforward NNs are universal approximators to, in principle, any kind of physical potential energy function of any dimensionality ${ }^{73,74}$. They are characterized by a consecutive data processing, directed from the input layer through one or more hidden layers to the output layer. Each layer consists of one or more nodes, called neurons, which make an interconnection between two neighboring layers by so-called weights. The weights are the arguments of the transfer or activation function, the value of which determines the numerical value of the interconnected next layer node. The subsequent application of this recipe over all 
layers and nodes yields a composed (preferentially) non-linear function for the NN topology given in Figure 1 of the form:

$V(\vec{G})=f\left(\sum_{j=1}^{3} w_{j 1}^{2} f\left(\sum_{i=1}^{2} w_{i j}^{1} g_{i}+a_{i}^{1}\right)+a_{j}^{2}\right)$.

In Eq.( 1$), \vec{G}$ is the input vector with the element $g_{j}, w_{i j}^{l}$ is the weight connecting the $j^{t h}$ node of layer $l$ with the $i$ th node of layer $l-1, f(x)=\tanh (x)$ is the activation function chosen here, and $a_{i}^{l}$ is a bias value which shifts the origin of the activation function.

The number of layers and nodes, and the interconnection structure specify the topology of the network and, therefore, its ability to adapt to the complexity of the problem at hand. For a chosen topology, the weights need to be adjusted to make the $\mathrm{NN}$ yield a desired output for a given input. This adjustment is called the training or the learning process, and involves a high-dimensional optimization procedure. Thus, the output of a network can be understood as the learned response $V$ of the network to a stimulus defined by the input vector $\vec{G}$.

We aim to represent a NN potential (NNP) as a function of the six adsorbate degrees of freedom of the $\mathrm{HCl}$ molecule on $\mathrm{Au}(111)$. The coordinates are the center of mass (COM) position $(X, Y, Z)$ of $\mathrm{HCl}$ relative to a gold atom, where $X$ and $Y$ determine the lateral position of the COM, and $Z$ is the molecule-surface distance. The $\mathrm{H}-\mathrm{Cl}$ bond length is denoted by $r$ and the relative orientation of the molecule is represented by the polar angle $\theta$ and the azimuthal angle $\phi$ (see Figure 2). 


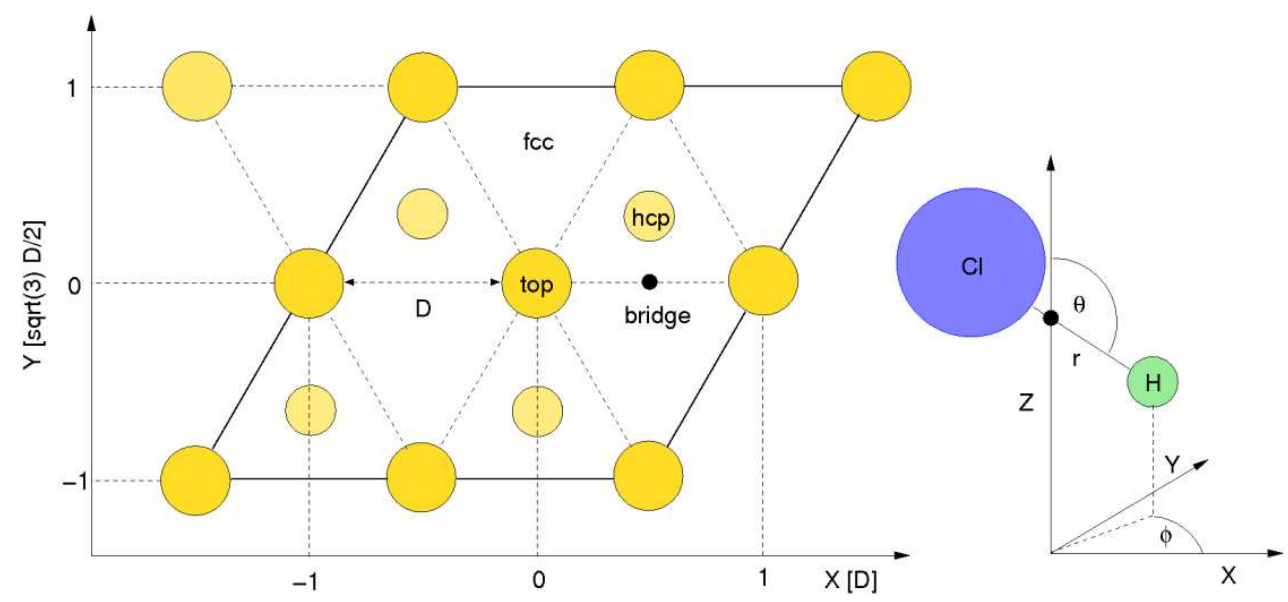

Figure 2: Left, top view of the $\mathrm{Au}(111)$ surface. Indicated are the surface lattice constant $D$ and the four highsymmetrical adsorption sites (top, bridge, hcp, fcc). Right, coordinate system with the six molecular coordinates of HCl.

In addition to the training procedure and the choice of the topology, one also needs to take into account symmetry when working with NNs. This concerns, for example, the invariance of the PES if chemically equivalent atoms are exchanged, or if symmetry equivalent coordinates exist. For molecule-surface systems dealt with within the rigid surface approximation, Behler et al. ${ }^{75}$ suggested the use of symmetry-adapted input coordinates rather than molecular or Cartesian coordinates. This modification has been later refined in Ref. $^{76}$ and connected to the formalism of permutationally invariant polynomials in Ref. ${ }^{77}$. For our system, consisting of a heteronuclear diatomic molecule interacting with a rigid surface of hexagonal structure, we use transformation functions, similar to the ones given in Ref. $^{78}$ :

$$
\begin{aligned}
& g_{1}\left(x_{H}, y_{H}, z_{H}\right)=e^{-\frac{1}{2} z_{H}} h\left(x_{H}-\frac{1}{4} D, y_{H}-\frac{1}{4 \sqrt{3}} D\right), \\
& g_{2}\left(x_{H}, y_{H}, z_{H}\right)=e^{-\frac{1}{2} z_{H}} h\left(x_{H}+\frac{1}{4} D, y_{H}+\frac{1}{4 \sqrt{3}} D\right), \\
& g_{3}\left(z_{H}\right)=e^{-\frac{1}{2} z_{H}}, \\
& g_{4}\left(x_{C l}, y_{C l}, z_{C l}\right)=e^{-\frac{1}{2} z_{C l}} h\left(x_{C l}-\frac{1}{4} D, y_{C l}-\frac{1}{4 \sqrt{3}} D\right),
\end{aligned}
$$




$$
\begin{aligned}
& g_{5}\left(x_{C l}, y_{C l}, z_{C l}\right)=e^{-\frac{1}{2} z_{C l}} h\left(x_{C l}+\frac{1}{4} D, y_{C l}+\frac{1}{4 \sqrt{3}} D\right), \\
& g_{6}\left(z_{C l}\right)=e^{-\frac{1}{2} z_{C l}}, \\
& g_{7}(X, Y, Z)=e^{-\frac{1}{2} Z} h\left(X-\frac{1}{4} D, Y-\frac{1}{4 \sqrt{3}} D\right), \\
& g_{8}(X, Y, Z)=e^{-\frac{1}{2} Z} h\left(X+\frac{1}{4} D, Y+\frac{1}{4 \sqrt{3}} D\right), \\
& g_{9}(Z)=e^{-\frac{1}{2} Z}, \\
& g_{10}(r)=r .
\end{aligned}
$$

One can easily see that the original six coordinates are over-defined by the ten-dimensional input vector $\vec{G}$. This is to account for the symmetry of the surface, for which a modified symmetry-adapted function:

$$
h(x, y)=\frac{1}{3 \sqrt{3}}\left(\sin \left(\frac{2 \pi}{D}\left(x-\frac{y}{\sqrt{3}}\right)\right)+\sin \left(\frac{2 \pi}{D} \frac{2 y}{\sqrt{3}}\right)+\sin \left(\frac{2 \pi}{D}\left(x+\frac{y}{\sqrt{3}}\right)\right)+\frac{3 \sqrt{3}}{2}\right)
$$

is employed. Here, $h(x, y)$ is defined on an interval $[0,1]$ and is chosen in accordance with the $A_{1}$-irreducible representation of the point group $C_{3 v}$ and the periodicity of the surface. More details are given in Ref. ${ }^{78}$. We further note, that the functions $g_{1}-g_{9}$ are damped along the atom-surface and the molecule-surface distances, respectively. This leaves $\vec{G}$ exclusively dependent on $r$ at positions in the gas phase, and the same is true for the potential.

For the training procedure, we prepare a set of 157,452 DFT potential points, which we divide into a training set with 141,707 points, and a reference set with 15,745 points. The latter set is used to determine the root mean square (RMS) error of the NNP on points not included in the fitting. We chose a 10-30-30-1 NN topology, that is, one input layer consisting of 10 nodes, two hidden layers with 30 nodes each and, finally, a single output node. After 100 training 
iterations, the NNP shows an RMS error of $12.4 \mathrm{meV}$ on the training set and $19.5 \mathrm{meV}$ on the reference set. The parameters of the extended Kalman filter described in Ref. ${ }^{79}$ and references therein are $\lambda(0)=0.985$ and $\lambda_{0}=0.9985$, and the factor for the adaptive filtering is set to $a_{t h}=0.3$

\subsection{Dynamical simulations}

\subsubsection{MD and MDEF simulations}

Reaction probabilities for $\mathrm{HCl}$ on $\mathrm{Au}(111)$ are determined with MD simulations and with $\mathrm{MD}$ simulations with electronic friction (MDEF). For both, we perform dynamics calculations on the 6D NNP described in Sec. 2.2 and neglect energy dissipation from the $\mathrm{HCl}$ molecule to the gold surface through phonon excitation. While an electronically adiabatic process is assumed in the MD simulations, we also allow for non-adiabatic effects in the MDEF simulations, that is, a coupled electron-nuclei dynamics due to the creation of electron-hole pairs in the metal surface. In this work, energy dissipation is described phenomenologically by coupling a $N$-dimensional system (molecule) to a heat bath (electrons in the metal surface) via an $(N \times N)$ frictional tensor $\underline{\underline{\eta}}^{80}$. The force $F_{i}$ that acts along a generalized coordinate $q_{i}$ of the molecule associated with the mass $m_{i}$ is then given by a Langevin equation:

$F_{i}=m_{i} \frac{d^{2} q_{i}}{d t^{2}}=-\frac{\partial V}{\partial q_{i}}-\sum_{k=1}^{N} \eta_{i k} \frac{d q_{i}}{d t}+R_{i}(T)$

Here, the first term on the rhs describes the force resulting from the PES, the second term is the frictional force resulting from the tensor elements $\eta_{i j}$, and the third term is a random force that arises from a finite bath with temperature $T$, which we assume to be the electronic temperature $T_{e l}$ in the following. In accordance with the second fluctuation-dissipation theorem $^{81}$, the random forces are calculated as: 
$R_{i}(T)=\sqrt{2 k_{B} T \eta_{i i} / \Delta t} \varepsilon$,

with $k_{B}$ being the Boltzmann constant and $\varepsilon$ a random number simulating a Gaussian white noise distribution, which we calculate by using the Box-Muller algorithm ${ }^{82}$. At $T=0 \mathrm{~K}$, the random forces in Eq.( 13 ) vanish, that is, heat transfer from the surface to the molecule is prohibited. The frictional term, however, remains, which leads to a loss of energy in the molecular DOF once the frictional forces become effective in the reactive process.

We use a velocity-Verlet based stochastic integrator developed by Ermak and Buckholtz ${ }^{83}$ to solve Eq.( 13 ). In the electronically adiabatic case $(\eta=0)$, the Langevin equation obeys Newton's equation of motion (NEM), which we solve in the MD simulations. The integrator then becomes the non-stochastic velocity-Verlet algorithm, which is known to work well in this regime.

For both MD and MDEF simulations we use a time step $\Delta t$ of about $10 \hbar / E_{h}(\approx 0.25 f s)$ which allows, on average, for an error in the energy conservation of less than $1 \mathrm{meV}$ in the MD simulations. The maximum error found is below $15 \mathrm{meV}$. Convergence test performed for MDEF simulations showed that the results are not affected by the size of the time step in the range from $0.5 \hbar / E_{h}(\approx 0.012 f s)$ to $10 \hbar / E_{h}(\approx 0.25 f s)$. For each set of initial conditions, we calculated 50,000 trajectories starting with the molecule at a distance of $Z=7.0 \AA$ to the surface and approaching the surface. A trajectory is considered to result in dissociative adsorption if the interatomic distance $r$ becomes larger than $2.58 \AA$. Trajectories are counted as scattered if the molecule-surface distance $Z$ becomes greater than $7.0 \AA$, and molecules are moving away from the surface. 


\subsubsection{AIMD simulations}

We perform AIMD simulations using the PBE and RPBE functionals, and the slab model as explained in Sec. 2.1. We chose a time step of $0.5 f s$ at which the error in the energy conservation is on average about $10 \mathrm{meV}$, with a maximum error of $41 \mathrm{meV}$ considering all trajectories. Depending on the incidence translational energy, we calculate a total of 300-500 trajectories per energy point, ensuring a standard error lower than 0.03 in the reaction probabilities. All trajectories start at $Z=7.0 \AA$. We apply the same conditions for assigning trajectories as adsorbed or scattered as in the $\mathrm{MD}(\mathrm{EF})$ simulations, see above.

With the help of AIMD simulations, we are also able to investigate the effect of the surface temperature $T_{S}$ as well as of energy dissipation to the surface phonons on the dissociation of $\mathrm{HCl}$ on $\mathrm{Au}(111)$. The process is, however, considered to proceed adiabatically on the electronic ground state potential, and ehp excitations are not accounted for. Simulations were performed for the following three different cases: i) the surface atoms remain fixed at their initial equilibrium positions during the dynamics (the Born-Oppenheimer static surface (BOSS) model) ii) the surface atoms are initially stationary at their equilibrium positions without having initially kinetic energy, but are allowed to move during the reaction (the $T_{S}=0 \mathrm{~K}$ case), and iii) the surface atoms are thermalized according to a surface temperature of $T_{S}=298 \mathrm{~K}$, and their motion is coupled to the motions of all the other surface atoms and the impinging $\mathrm{HCl}$ molecule.

To simulate a thermalized surface, we prepare an ensemble of 20,000 slab configurations, each of which is a representative of a microscopic phase space configuration at a target temperature $T_{S}$. The chosen thermalization procedure requires the following steps (see also Ref. $\left.^{84}\right)$ : 
1.) Expansion of the surface lattice constant $D$ according to the thermal expansion of gold at $T_{S}$, while the interlayer distances $d_{12}, d_{23}$ and $d_{34}$ (see above) remain unchanged.

2.) Initialization of 20 slab models with random displacements and velocities according to $T_{S}=298 \mathrm{~K}$ followed by an NVT simulation (number of particles, volume and temperature are kept constant) for each slab, 1 ps long.

3.) Final performance of NVE simulations (number of particles, volume and energy are kept constant) for all slabs, each slab for another 2 ps. During the second half of these simulations (last $1 p s$ ), snapshots of the slab are taken at every time step ( $1 f s$ ) which serve as possible initial configurations of the thermalized slab in the corresponding AIMD simulations.

To expand $D$, we calculate the expansion factor $\xi$ by integrating the equation

$\xi=\exp \left(\int_{T=0 K}^{T_{S}} \kappa(T) d T\right)$

numerically. The expansion coefficients $\kappa(T)$ were taken from experiments ${ }^{85}$. We obtain $\xi=1.0031668$, which yields $D\left(T_{S}=298 K\right)=2.948 \AA$ for the PBE functional. In order to prepare initial slab configurations at the targeted $T_{S}$, the initial velocities of the surface atoms are chosen randomly according to a Maxwell-Boltzmann distribution and their initial positions are obtained by randomly displacing the atoms from their equilibrium positions according to a Gaussian distribution of width

$\sigma_{k, i}=\sqrt{\frac{k_{B} T_{S}}{\left(2 \pi c \widetilde{v}_{k, i}\right)^{2} m_{A u}}}$

where $c$ is the speed of light in vacuum, $m_{A u}=196.966 \mathrm{amu}$ is the mass of a gold atom, and $\tilde{v}_{k, i}$ is the vibrational wavenumber of a single gold atom in layer $k$ vibrating along the $i^{\text {th }}$ normal mode vector. The vibrational wavenumbers are obtained by a normal mode analysis of a single $\mathrm{Au}$ atom in each layer. The corresponding values are listed in Table 1. We allow for a 
maximum initial displacement from the equilibrium position of $\pm 0.125 \AA$ directed along the respective normal mode vector. The thermalization procedure is applied to all layers, except the $4^{\text {th }}$ layer, which we kept fixed. From the total energies of the 20 slab configurations obtained from the NVT calculations, we compute the resulting $T_{S}$ to be $330 \mathrm{~K} \pm 23 \mathrm{~K}$.

Table 1: Vibrational wavenumbers for a single gold atom in the different layers of the slab model at $T_{S}=0 \mathrm{~K}$, see text. The $1^{\text {st }}$ layer is the uppermost layer. The $x, y, z$ specification of the wavenumbers indicates the largest Cartesian component of the corresponding normal mode vector. The results are taken from three different frequency analyses using VASP.

\begin{tabular}{|c|c|c|c|}
\hline & $\tilde{\boldsymbol{v}}_{\boldsymbol{x}}[\mathbf{1} / \mathbf{c m}]$ & $\tilde{\boldsymbol{v}}_{\boldsymbol{y}}[\mathbf{1} / \mathbf{c m}]$ & $\tilde{\boldsymbol{v}}_{\boldsymbol{z}}[\mathbf{1} / \mathbf{c m}]$ \\
\hline $\mathbf{1}^{\text {st }}$ layer & 77.05 & 77.27 & 67.75 \\
\hline $\mathbf{2}^{\text {nd }}$ layer & 87.64 & 86.38 & 86.38 \\
\hline $\mathbf{3}^{\text {rd }}$ layer & 87.36 & 87.40 & 87.82 \\
\hline
\end{tabular}

\subsubsection{Initial conditions}

In the following, we describe in detail the methodology used to setup the initial conditions in the dynamics. All results presented in this work are obtained from quasiclassical simulations, that is, the impinging molecules initially have an internal energy $E_{v, j, m}$ associated with the quantum mechanical rovibrational state characterized by the vibrational quantum number $v$, the rotational momentum quantum number $j$, and the magnetic rotational angular momentum quantum number $m_{j}$. The Hamilton operator for $\mathrm{HCl}$ in the gas phase and for given $j$-value reads:

$\widehat{H}_{j}(r)=-\frac{\hbar^{2}}{2 \mu} \frac{\partial^{2}}{\partial r^{2}}+V_{e f f}^{j}$

where $\hbar$ is Dirac's constant and $\mu=m_{H} m_{C l} /\left(m_{H}+m_{C l}\right)$ is the reduced mass of $\mathrm{HCl}$. The effective potential 
$V_{e f f}^{j}(r)=V_{g a s}(r)+\frac{\hbar^{2}}{2 \mu r^{2}} j(j+1)$

contains the gas phase potential $V_{\text {gas }}$, and the rotational energy term for a given quantum number $j$, both being functions of $r$. The rovibrational energies are obtained by diagonalizing the Hamilton matrix, represented in a discrete variable representation ${ }^{86}$ by using 128 grid points in $r$ on the interval $r \in[0.78,2.94] \AA$.

The classical probability distribution to find $\mathrm{HCl}$ in a configuration at certain $v, j, m_{j}$-values is sampled randomly. We first determine the time $t_{f}$ needed to complete a full vibrational period, if $\mathrm{HCl}$ moves on $V_{e f f}^{j}$ with an initial rovibrational energy $E_{v, j}$. Since $r$ is a welldefined function of time in a classical oscillator, we obtain the starting value of the trajectory in $r$ by a random selection of the initial time $t=\left[0, t_{f}\right]$. The angular distribution $P_{j, m_{j}}(\theta, \phi)$ for $j>0$ is obtained by first calculating the angle $\beta$ between the total angular momentum vector $\vec{J}$ and the space fixed $Z$-axis (pointing along the surface normal) using the relation $\cos (\beta)=m_{j} / \sqrt{j(j+1)}$, thereby accounting for the quantization of the angular momentum projected on the surface normal. We then randomly select the azimuthal orientation of $\vec{J}$ described by the angle $\alpha \in[0,2 \pi]$, and the angle $\gamma \in[0,2 \pi]$ that reflects the angular orientation of the $\mathrm{HCl}$ molecule in the plane perpendicular to $\vec{J}$. A coordinate transformation is then used to map the $\{\alpha, \beta, \gamma\}$-configuration onto the corresponding $\{\theta, \phi\}$-configuration. The resulting classical probability distributions are somewhat different from their quantum mechanical counterparts ${ }^{87}$ but they correctly describe the observation that a rotational state is also characterized by the alignment of the molecule relative to the surface. The procedure described is redundant for $j=0$, in which case we simply sample a $\sin (\theta)$-distribution and 
select a random $\phi$-value. The classical angular momenta are given by $l_{\phi}=\hbar m_{j}$ and $\left|l_{\theta}\right|=$ $\hbar \sqrt{j(j+1)-m_{j}^{2} / \sin ^{2}(\theta)}$.

In this work, we also study the effect of different beam distributions on the reaction probability $S$ of $\mathrm{HCl}$ on $\mathrm{Au}(111)$. We distinguish between reaction probabilities $S$ obtained for a beam i) with a monochromatic distribution of the translational energy and a well defined internal state, called $S_{\text {mono }}^{v, j, m_{j}}$, ii) with a translational energy distribution according to experiments, but with a well defined internal state, denoted as $S_{\text {trans }}^{v, j, m_{j}}$, and iii) with the translational energy and either the full rovibrational or the vibrational state population distribution taken according to the nozzle temperature $T_{n}$, denoted by $\bar{S}\left(T_{n}\right)$ and $\bar{S}^{j=0}\left(T_{n}\right)$, respectively, or with the experimentally determined rovibrational state population distribution, called $\bar{S}_{\text {exp }}\left(T_{n}\right)$ in the following. In the latter case, the rotational temperature $\left(T_{\text {rot }}\right)$ is different from the vibrational temperature $\left(T_{\text {vib }}=T_{n}\right)$. The two temperatures are approximately related to one another as follows:

$T_{\text {rot }}=-181.1 K+0.648 T_{v i b}$

(private communications).

The probability to find $\mathrm{HCl}$ in a certain rovibrational state and with a velocity $v$ in an interval $v+d v$ at a nozzle temperature $T_{n}$ is given by

$P\left(v, T_{n}\right) d v=A v^{3} e^{-\frac{\left(v-v_{0}\right)^{2}}{a^{2}}} f_{v, j}\left(T_{n}\right) d v$,

where $A$ is a normalization constant, $v_{0}=\sqrt{2 E_{0} / M_{H C l}}$ is the stream velocity and $a$ is the width of the distribution. The latter two parameters are tabulated for experiments on this system elsewhere $^{29}$. Eq. ( 20 ) defines a flux-weighted velocity distribution. The probability 
$f_{v, j}\left(T_{n}\right)$ to find the molecule in the state $v, j$ is, depending on the population distribution of interest, either given by

$f_{v, j}\left(T_{n}\right)=\frac{(2 j+1) e^{-\frac{E_{v, j}-E_{0,0}}{k_{B} T_{n}}}}{Z\left(T_{n}\right)}$,

if the rovibrational state is populated according to $T_{n}$, or

$f_{v, j}\left(T_{n}\right)=\frac{1}{Z\left(T_{n}\right)}(2 j+1) e^{-\frac{E_{v, 0}-E_{0,0}}{k_{B} T} v_{v i b}} e^{-\frac{E_{\nu, j}-E_{\nu, 0}}{k_{B} T_{r o t}}}$,

if the experimental conditions are taken fully into account. Here, $Z\left(T_{n}\right)$ is the partition function and is different for the two cases considered above. In all that follows, we consider normal incidence conditions, that is, $v_{X}=v_{Y}=0$.

\subsubsection{Calculation of observables}

Each trajectory represents the dynamical evolution of a microscopic state. We therefore calculate macroscopic observables $\langle U\rangle$ as the average of $M$ microscopic measurements $u_{i}$ :

$\langle U\rangle=\frac{1}{M} \sum_{i=1}^{M} u_{i}$.

Here, $\langle U\rangle$ can be the sticking probability $S$ for $\mathrm{HCl}$ on $\mathrm{Au}(111)$, in which case $M$ is the total number of calculated trajectories, and $u_{i}=1$ for dissociatively adsorbed and $u_{i}=0$ for scattered trajectories. The energy transfer from the molecule to the surface is also computed accordingly, where $u_{i}$ is then the energy difference of the initial and final slab configuration, and $M$ is the number of scattered AIMD trajectories. The average loss of energy due to ehp excitation is calculated similarly by averaging the loss of energy individually for all adsorbed and scattered MDEF trajectories, respectively. 


\subsection{Electronic friction model}

For the MDEF simulations, the friction coefficients for the $i^{\text {th }}$ atom are calculated in this work according to the local density friction approximation (LDFA) ${ }^{88}$. The LDFA was derived for atoms embedded in a homogeneous free electron gas which scatters on the symmetric Coulomb potential of the bare nuclei. The friction coefficient is then related to the phase shift $\Upsilon$ of the impinging and the scattered waves of the electrons as follows

$\eta_{i i}\left(\underline{r}_{i}\right)=\frac{3 \hbar}{r_{s}^{2}\left(\underline{r}_{i}\right)}\left(\frac{4}{9 \pi}\right)^{1 / 3} \sum_{l=0}^{\infty}(l+1) \sin ^{2}\left(Y_{l}^{i}\left(r_{s}, \varepsilon_{f}\right)-Y_{l+1}^{i}\left(r_{s}, \varepsilon_{f}\right)\right)$

Here, $Y_{l}^{i}\left(r_{s}, \varepsilon_{f}\right)$ is the phase shift of the $l^{\text {th }}$ partial wave at the Fermi energy $\varepsilon_{f}$ and is tabulated in Ref. $^{89}$ for different $l$-values and Wigner-Seitz radii:

$r_{s}\left(\underline{r}_{i}\right)=\left(\frac{3}{4 \pi \rho\left(\underline{r}_{i}\right)}\right)^{1 / 3}$

of the embedding electron density $\rho$. We assume that the embedding density to a good approximation corresponds to the electron density of the bare, frozen ideal Au(111) surface which we obtain from a single DFT calculation. (We expect this way of representing the electron density to be a good approximation for the low surface temperature of $170 \mathrm{~K}$ at which the molecular beam experiments were performed, and recent work by Novko et al. ${ }^{90}$ supports this view.) Since $\rho$ is a function of the position $\underline{r}_{i}=\left(x_{i}, y_{i}, z_{i}\right)^{T}$ of the impurity screening the surface, so is the friction coefficient. In the MDEF simulations, we do not explicitly evaluate Eq.( 24 ). Instead, we first calculate the friction coefficients for $\mathrm{H}$ and $\mathrm{Cl}$ according to Eq.( 24 ) at $r_{s}$-values for which phase shifts were given in Ref. ${ }^{89}$ and fit the results to the function

$\eta_{i i}=\operatorname{ar}_{s}^{b}\left(\underline{r}_{i}\right)\left(e^{-c r_{s}\left(\underline{r}_{i}\right)}\right)^{d}$ 
where the parameters for hydrogen are $a=0.70881 \hbar / a_{0}^{b+2}, b=0.54188, c=$ $0.68314 a_{0}^{-1}$ and $d=1.0$. For the chlorine atom, we obtain $a=16.7532 \hbar / a_{0}^{b+2}, b=$ 1.11661, $c=1.31171 a_{0}^{-1}$ and $d=1.3117$. Note that the friction coefficients are in principle different for $\mathrm{H}$ and $\mathrm{Cl}$ at the same electron density due to the different phase shifts $Y_{l}^{i}$ for $i=\mathrm{H}, \mathrm{Cl}$

The LDFA formalism outlined above uses the independent atom approximation (LDFA-IAA) and describes a spherically symmetric problem. Molecular properties are completely neglected, and as a result the friction coefficients are directionally independent, i.e., the atom will feel the same local friction along its three DOF. As a consequence, the LDFA may not allow for the computation of intermode couplings between different DOF of the molecule, and the non-diagonal elements $(i \neq k)$ of the friction tensor $\eta_{i k}$ are zero. Other methods have been proposed to circumvent these limitations ${ }^{90-93}$. Nonetheless, the LDFA has been successfully combined with MD simulations to describe, for example, the laser-induced desorption process of $\mathrm{H}_{2}$ from $\mathrm{Ru}(0001)^{94,95}$. In spite of the present drawbacks of the model, the LDFA has considerable advantages, particularly in the context of MDEF simulations. It is in principle a parameter-free model (although a fitted expression for the friction is used in this work) and, therefore, reproducible since phase shifts and electron densities are computed with non-empirical methods. Additionally, friction coefficients are easily calculated, and are obtained directly as functions of atomic Cartesian coordinates. This makes the LDFA-MDEF scheme particularly suitable for the stochastic integrator scheme we use to solve the Langevin equation as described in Sec. 2.3.1. Finally, although the LDFA method was originally developed to describe the stopping power of bulk metals for bare ions such as $\mathrm{H}^{+}{ }^{96}$, meanwhile the theory has been used successfully to describe energy loss to electron-hole pair excitation in scattering of neutral atoms from metal surfaces, for instance in the $\mathrm{H}+\mathrm{Au}(111)$ 
system $^{8}$. This is perhaps not surprising: The calculation of the phase shifts is done for an impurity (say the atomic nucleus of $\mathrm{H}$ ) embedded in a homogeneous electron gas, but in this actual system $\mathrm{H}$ is best described as $\mathrm{H}^{-97}$, and not as $\mathrm{H}^{+}$or $\mathrm{H}$ with the methodology used in Ref. $^{89}$.

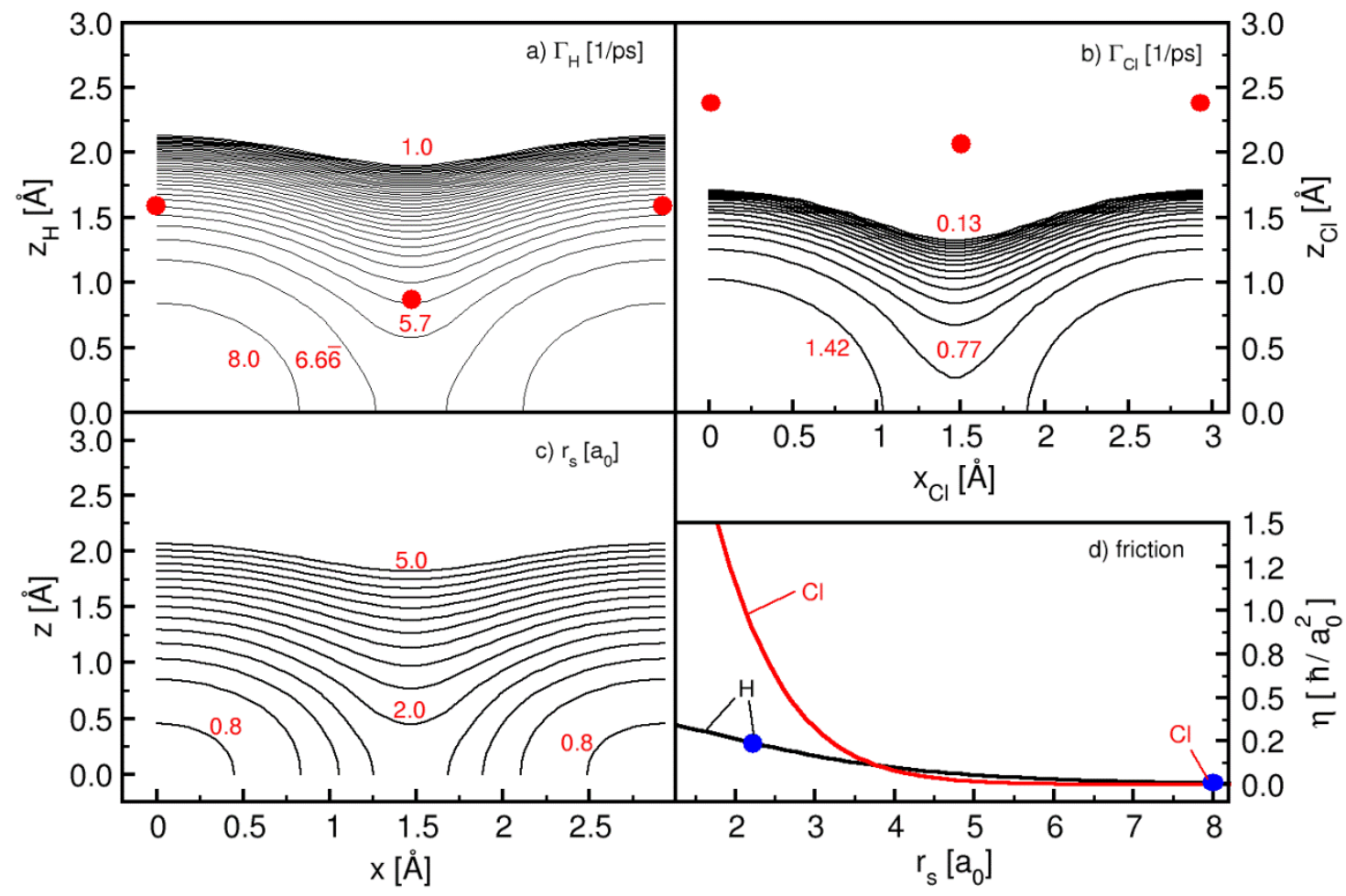

Figure 3: Depiction of the dissipative rate $\Gamma$ in $1 / p s$ as function of the $x$ and $z$ coordinates $(y=0)$ for a) hydrogen and b) chlorine on $\mathrm{Au}(111)$ according to the fitted LDFA friction model. The red dots in a) and b) indicate the equilibrium atom-surface distances of $\mathrm{H}$ and $\mathrm{Cl}$ at the top and the bridge sites. Panel c) displays the Wigner-Seitz radius $\boldsymbol{r}_{s}$ of the electron density of the bare $\mathrm{Au}(111)$ also as function of $x$ and $z(y=0)$. In panel d), friction coefficients calculated according to Eq.( 26 ) are plotted as function of $r_{s}$ for $\mathrm{H}$ (black) and $\mathrm{Cl}$ (red). The blue points in d) indicate the $r_{s}$ and $\eta$-values for $\mathrm{H}$ and $\mathrm{Cl}$ at the TS geometry obtained with PBE. Contour plots are made with a level spacing of a) (25 $f s)^{-1}$, b) $(600 f s)^{-1}$ and c) $0.3 a_{0}$.

Related to $\eta$ is the (mass-independent) dissipative rate $\Gamma=\eta / m$, which is a more useful measure of the strength of the dissipative coupling between the system and the bath than the friction coefficient itself. In Figure $3 \mathrm{a}$ to $3 \mathrm{c}$, we plot the Wigner-Seitz radius $r_{s}$ of the electron density of the bare $\mathrm{Au}(111)$ surface and the dissipative rates as functions of the $x, z$ positions $(y=0)$ of the $\mathrm{H}$ and the $\mathrm{Cl}$ atom on $\mathrm{Au}(111)$. As can be seen, the $\Gamma$-plots in Figure $3 \mathrm{a}$ and $3 \mathrm{~b}$ 
reflect the corrugation of the surface. Large electron densities (small $r_{s}$-values) are found close to the surface atoms and the dissipative coupling is large at these positions. Since the electron density decays approximately with $1 / z$, the dissipative interaction decreases accordingly. The $\mathrm{H}$ and $\mathrm{D}$ atoms have to be close to the surface to make the non-adiabatic coupling act efficiently on the dynamics. In the current case, this appears to be for atomsurface distances smaller than $2 \AA$. We also note that the hydrogen atom interacts much more strongly with the surface electrons than the chlorine atom. This is due, firstly, to a much larger dissipative rate at similar atom-surface distances, although the friction coefficients are smaller for $\mathrm{H}$ than for $\mathrm{Cl}$ (see Figure 3d), and, secondly, to hydrogen penetrating deeper into areas associated with larger electron densities. To make this visible, we indicate the equilibrium distance $z_{e q}$ for $\mathrm{H}$ and $\mathrm{Cl}$ at the top and the bridge sites by red dots in Figure 3a and 3b. As can be seen, the $z_{e q}$-values for $\mathrm{Cl}$ are so large that the dissipative rate is low, as surmised from Figure $3 \mathrm{~d}$ where $\eta$ is given as function of $r_{s}$. The blue dots indicate the value of the friction coefficient of $\mathrm{H}$ and $\mathrm{Cl}$ at the TS geometry calculated with PBE. These observations suggest that the electronically non-adiabatic energy flow between the $\mathrm{HCl}$ molecule and the $\mathrm{Au}(111)$ surface is most likely mediated by the hydrogen atom in the LDFA description.

\section{Results}

\subsection{Electronic structure results and PES}

In the following, we have a closer look into some features of the 6D NNP and the potential along the reaction coordinate for $\mathrm{HCl}$ on an unreconstructed $\mathrm{Au}(111)$ surface. In Figure 4, we first present nudged elastic band (NEB) calculations ${ }^{98,99}$ employing the PBE and RPBE functionals, together with results taken from $\mathrm{Ref}^{43}$ for $\mathrm{HCl}$ approaching the surface from the gas phase and arriving at its adsorbed global minimum geometry. As can be seen, the 
adsorption of $\mathrm{HCl}$ is predicted to be an endothermic process. The product states found with PBE and PW91 on the one hand and with RPBE on the other hand are about $400 \mathrm{meV}$ and $700 \mathrm{meV}$ higher in energy than the reactant state. In Ref. ${ }^{43}$, the most stable product state was found for $\mathrm{H}$ and $\mathrm{Cl}$ adsorbed at two adjacent $f c c$ sites. PBE yields very similar results. With RPBE, however, we find that the most stable geometry is obtained if $\mathrm{H}$ and $\mathrm{Cl}$ form a layer with a regular hexagonal structure, in which three $\mathrm{Cl}$ atoms (including the periodic images) almost equidistantly surround an $\mathrm{H}$ atom. Hydrogen adsorbs then at the $f c c$ site and $\mathrm{Cl}$ at the second nearest top site.

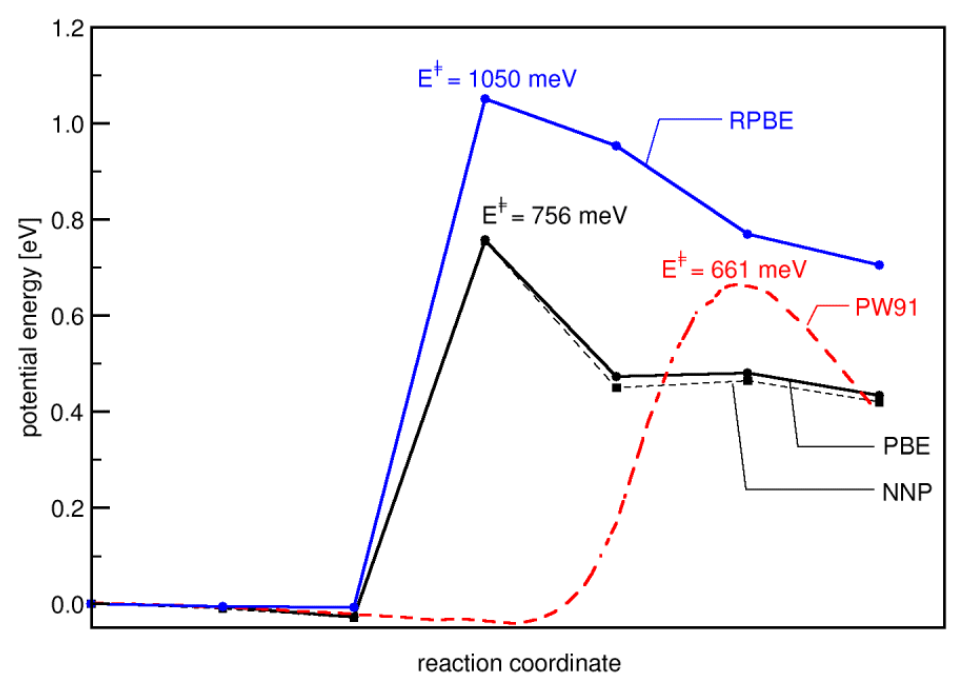

Figure 4: Potential energies along the reaction coordinate obtained by NEB calculations employing the PBE (black solid line) and RPBE (blue solid line) functionals. For comparison, also plotted are PW91 results taken from Ref. ${ }^{43}$ (red dashed line) and the NNP values for geometries obtained with the PBE-NEB calculations. The transition state for the RPBE has been obtained by an additional refined search using the DIMER method ${ }^{100-103}$.

The transition state energies lie in a comparably wide range of energies. PW91 and PBE yield minimum barrier heights of $661 \mathrm{meV}$ and $756 \mathrm{meV}$, respectively (see Table 2). This is not as similar as expected for these functionals. The potential values given in Ref. ${ }^{43}$ seem not to be well converged with respect to the number of k-points used. In Ref. ${ }^{43}$, the used Monkhorst grid pack was $5 \times 5 \times 1$, here we use $11 \times 11 \times 1$ - this is the major difference in the computational 
setups. Using the same $\mathrm{HCl}$ geometries at the TS and the gas phase as obtained by PBE, we compute for the PW91 functional a barrier of $669 \mathrm{meV}$ with $5 \times 5 \times 1$ and $745 \mathrm{meV}$ with $11 \times 11 \times 1$ in much better agreement with the PBE value of $756 \mathrm{meV}$. With RPBE, we obtain a considerably larger value of $1050 \mathrm{meV}$. (The barrier heights appear to be well converged with respect to the surface unit cell size to within $20 \mathrm{meV}$, as may be seen by comparing the $(3 \times 3)$ to the $(2 \times 2)$ surface unit cell $(\mathrm{R}) \mathrm{PBE}$ results in Table 2$)$. The geometry calculated with PBE is shown in Figure 5 and described in Table 2. The $\mathrm{H}$ atom points towards the surface and the $\mathrm{HCl}$ bond makes an angle of $\theta=132.9^{\circ}$ with the surface normal, and $\phi$ is approximately $330^{\circ}$. The $\mathrm{H}-\mathrm{Cl}$ distance is $r=1.934 \AA$ at the barrier, and the barrier to dissociation may therefore be classified as late (the gas phase value being $1.27 \AA$ ). The COM position is close to the $t 2 f$ site with $Z=2.398 \AA$. These values are in good agreement with previous findings of Zhang and coworkers ${ }^{43}$, who similarly found the COM of the TS approaching near the $f c c$ site.
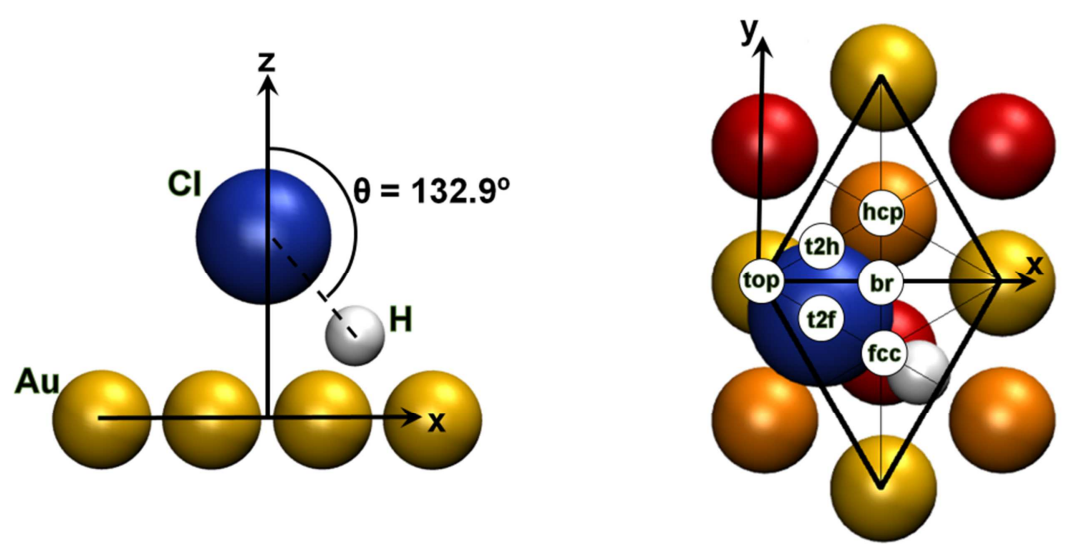

Figure 5: Transition state geometry of $\mathrm{HCl}$ on $\mathrm{Au}(111)$ taken from a NEB calculation using the PBE functional, side view (left) and top view (right). Indicated are also the different high-symmetric adsorption sites. Chlorine is represented in blue, hydrogen in white, the $1^{\text {st }}$ Au layer in gold, the $2^{\text {nd }}$ layer in orange and the $3^{\text {rd }}$ layer in red.

The value of $\theta=132.9^{\circ}$ is interesting and may reflect the different atom sizes of $\mathrm{H}$ and $\mathrm{Cl}$. Assuming, for example, a flat surface and two hard spheres with empirical atomic radii of 100 
$p m$ for $\mathrm{Cl}$ and $25 \mathrm{pm}$ for $\mathrm{H}$, we find that $\theta$ must be at least $113^{\circ}$ at the $r$-value of the TS. Although the RPBE barrier is much higher, the geometry of the TS calculated with RPBE (and a refined search with the DIMER method ${ }^{100-103}$ ) is very similar to the PBE-TS geometry shown in Figure 5. The $\mathrm{H}-\mathrm{Cl}$ bond length is $r=1.947 \AA$, the polar angle $\theta=134.8^{\circ}$ and $Z=2.435 \AA$. The TSs we calculated are all $1^{\text {st }}$ order saddle points which we verified by a normal mode analysis. From the NEB calculations, we also notice an attractive well of $30 \mathrm{meV}$ for PBE and $7 \mathrm{meV}$ for RPBE in the entrance channel in front of the barrier. This, however, should not be interpreted as a van der Waals well, since neither of the functional accounts for the attractive London dispersion interaction. Experiments estimate the orientationally averaged well depth to be about $220 \mathrm{meV}^{35}$.

Table 2: Listed are the transition state energies $E^{\ddagger}$, the molecule-surface distance $Z^{\ddagger}$, the interatomic distance $r^{\ddagger}$ and the polar orientation $\boldsymbol{\theta}^{\ddagger}$ for $\mathrm{HCl}$ on $\mathrm{Au}(\mathbf{1 1 1})$ at the TSs calculated with different DFT functionals in this and in previous works $^{43,104}$. The barrier heights listed in brackets are given for single point calculations employing a (3×3) supercell and a $9 \times 9 \times 1$ k-point sampling, but for the geometries that were taken from $(2 \times 2)$ NEB/DIMER calculations.

\begin{tabular}{|l|c|c|c|c|}
\hline & $\boldsymbol{r}^{\ddagger}[\AA]$ & $\boldsymbol{Z}^{\ddagger}[\AA]$ & $\boldsymbol{\theta}^{\ddagger}\left[{ }^{\circ}\right]$ & $\boldsymbol{E}^{\ddagger}[\mathbf{m e V}]$ \\
\hline RPBE, this work & 1.947 & 2.435 & 134.8 & $1050(1071)$ \\
\hline RPBE, Ref. $^{\mathbf{1 0 4}}$ & 2.100 & - & 155.0 & 910 \\
\hline PBE, this work & 1.934 & 2.398 & 132.9 & $756(773)$ \\
\hline${\text { PW91, } \text { Ref. }^{\text {43 }}}$ & 1.917 & 2.394 & 131.7 & 661 \\
\hline
\end{tabular}

Before discussing a few properties of the 6D NNP (based on PBE) in detail, we first mention the excellent accuracy of the fit. In Figure 4, we compare potential values taken from PBENEB calculations and the corresponding NNP values obtained for the NEB geometries. In the entrance channel and at the barrier, the NNP deviates from the DFT points by less than $4 \mathrm{meV}$, and for geometries near the product state by only $13 \mathrm{meV}$ to $23 \mathrm{meV}$. These results are promising, and indicate that the fit is suitable for describing the dynamics of $\mathrm{HCl}$ on a 
rigid $\mathrm{Au}(111)$ surface, which we will confirm below in our comparison of MD and AIMD results.

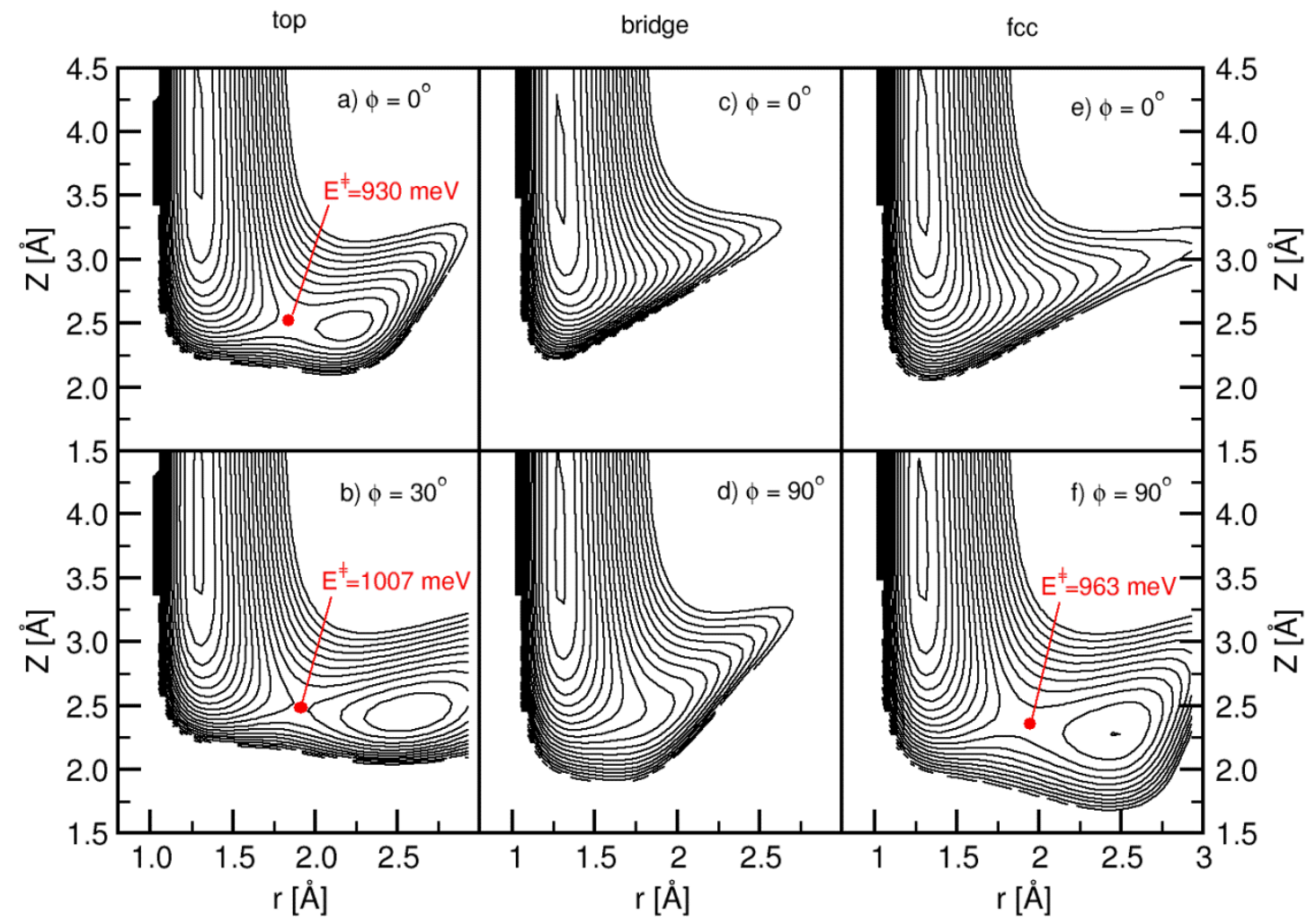

Figure 6: Two-dimensional potential cuts of the NNP for $\mathrm{HCl}$ on a rigid Au(111) along $r$ and $Z$ for the COM located at different adsorption sites and for different $\phi$-orientations of the molecule. All cuts are made for $\theta=133^{\circ}$. Indicated are also the late barriers on several cuts and their potential values. The contour level is $0.1 \mathrm{eV}$ for an energy range of $[0,1.9] e V$.

In Figure 6, we present a few contour plots of two-dimensional (2D) NNP cuts as function of the $r$ and $Z$ coordinates for the COM located at the top, bridge and $f c c$ sites, and at different azimuthal orientations of the molecule. The polar angle is fixed at $\theta=133^{\circ}$ for all cuts, in accordance with the value of the TS. The 2D PESs have a typical elbow form and show adsorption wells that appear as plateaus for the reactive configurations at large interatomic distances $(r>2 \AA$, see Figure 6a, 6b and 6f) We find second barriers in the plots showing reactive configurations which might appear to block the dissociation towards even larger distances $r$. However, this is an artifact of keeping the impact orientation angle $\theta$ fixed at the 
value of $133^{\circ}$ characterizing the TS, thereby placing the hydrogen atom into the unfavorable situation of penetrating the surface at large $r$-values. Nonetheless, in the dynamics the shape noted of the PES for fixed polar angle lead to back reactions (and we have indeed seen this in the AIMD simulations) unless the molecule is able to reorient along $\theta$ once it crosses the TS.

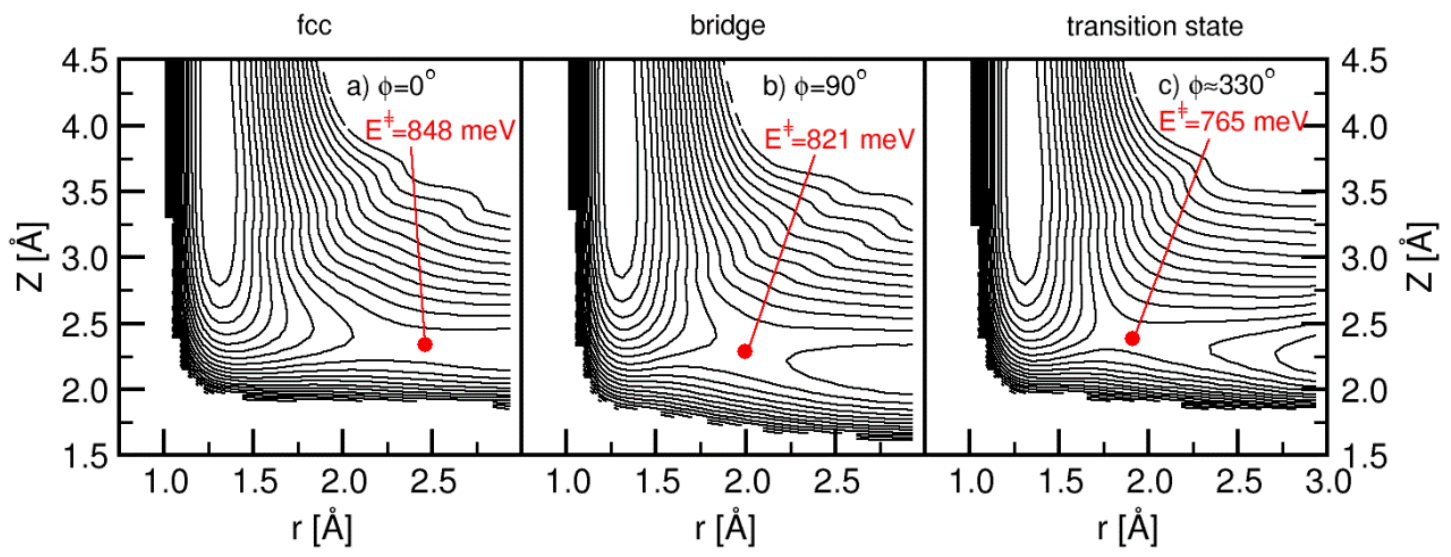

Figure 7: Contour plots of 2D NNPs for $\mathrm{HCl}$ approaching the $\mathrm{Au}(111)$ surface at a) the $f c c$ site with $\phi=0^{\circ}$, b) the bridge site with $\phi=90^{\circ}$, and c) with the COM located at the TS obtained from the NEB calculations. In all cases, the polar angle $\theta$ is optimized. The contour spacing is $0.1 \mathrm{eV}$ for an energy range of $[0,1.9] \mathrm{eV}$.

Reorientation effects may play a particularly important role at impact sites that are seemingly unreactive. For example, the potential cut at the top site in Figure 6a is reactive, but at the bridge and the $f c c$ positions the 2D PESs are not (Figure 6c to 6e). If we, however, allow for a relaxation along $\theta$, these potential cuts become reactive, too. To illustrate this, we show 2D cuts in Figure 7 for $\mathrm{HCl}$ approaching the surface at the $f c c$ and bridge sites in a similar way as shown in Figure 6d and 6e, and with $X, Y, \phi$ coordinates equal to the TS geometry found with the NEB, but now with optimized $\theta$-values. At the $f c c$-barrier shown in Figure $7 \mathrm{a}, \mathrm{HCl}$ makes an angle $\theta$ of $114^{\circ}$, which becomes smaller as the dissociation proceeds. The barrier of 850 $m e V$ occurs at a larger value of $r(2.421 \AA$ ). Likewise, at the bridge site $\mathrm{HCl}$ impacts with a favorable orientation of $\theta=116^{\circ}$ at the barrier. The molecule-surface distance is, however, similar to that of the TS geometry. These results suggest that a reorientation dynamics needs 
to take place during the bond cleavage of $\mathrm{HCl}$, but also that most of the barriers lying between $750 \mathrm{meV}$ to $1000 \mathrm{meV}$ are accessible if the molecule impacts with orientations $\theta \epsilon[110,135]^{\circ}$.

\subsection{Dynamics results}

\subsubsection{AIMD results}

In Figure 8a, we plot reaction probabilities computed with the PBE functional as a function of the incident translational energy for $\mathrm{HCl}$ in the rovibrational ground state $(v=0, j=0)$. We apply three different models to investigate the effect of surface motion and assume a monochromatic translational energy distribution. The black curve represents the BOSS model (rigid surface), in blue are results for the surface initially at $T_{S}=0 \mathrm{~K}$, and in red for a thermalized surface at $T_{S}=298 \mathrm{~K}$ (the experimental surface temperature was $T_{S}=170 \mathrm{~K}^{29}$ ). In all cases the reaction probability $S$ increases with the translational energy from about 0.38 to about 0.55 in the energy range $1.4-2.0 \mathrm{eV}$. We further observe that the inclusion of surface atom motion reduces the absolute reactivity by only about $0.03-0.05$. For example, at $E_{\text {trans }}=1.6 \mathrm{eV}$ we obtain $S=0.48$ within the BOSS model, whereas for $T_{S}=0 \mathrm{~K}, S=$ 0.44. Keeping in mind that the standard deviation of the AIMD results is about \pm 0.025 , we can conclude that surface atom motion does not have a considerable effect on the reaction probability of $\mathrm{HCl}$ on $\mathrm{Au}(111)$.

The observed small effect of allowing surface motion may come as a surprise, since the Au$\mathrm{HCl}$ system shows a mass ratio that is indicative of the possibility of large amounts of energy being transferred from the incident molecule to the surface atom motion. As also noted in Ref. $^{28}$, phonon-mediated energy dissipation might then efficiently inhibit reaction. In Table 3, 
we list our AIMD results together with the amount of energy that is transferred from the molecule to the surface atoms computed by evaluating the energy additionally left in the $(2 \times 2)$ supercell after non-reactive scattering events. About $30 \%$ of the initial translational energy of the scattered molecules is dissipated to surface atom motion. This amount is almost independent of the surface temperature and the incidence translational energies. Using the Baule model ${ }^{105}$, we can estimate the energy transfer from $\mathrm{HCl}$ to a gold atom in the limit of two colliding hard spheres

$E_{\text {loss }}^{\text {Baule }}=E_{\text {trans }} \frac{4\left(M_{H C l} / m_{A u}\right)}{\left(1+M_{H C l} / m_{A u}\right)^{2}}$

where $m_{A u}$ is the mass of a gold atom, and $M_{H C l}$ the molecular mass of $\mathrm{HCl}$. The Baule model suggests an energy transfer of about $50 \%$ of the incidence translational energies (see Table 3 ), significantly larger than found with AIMD.

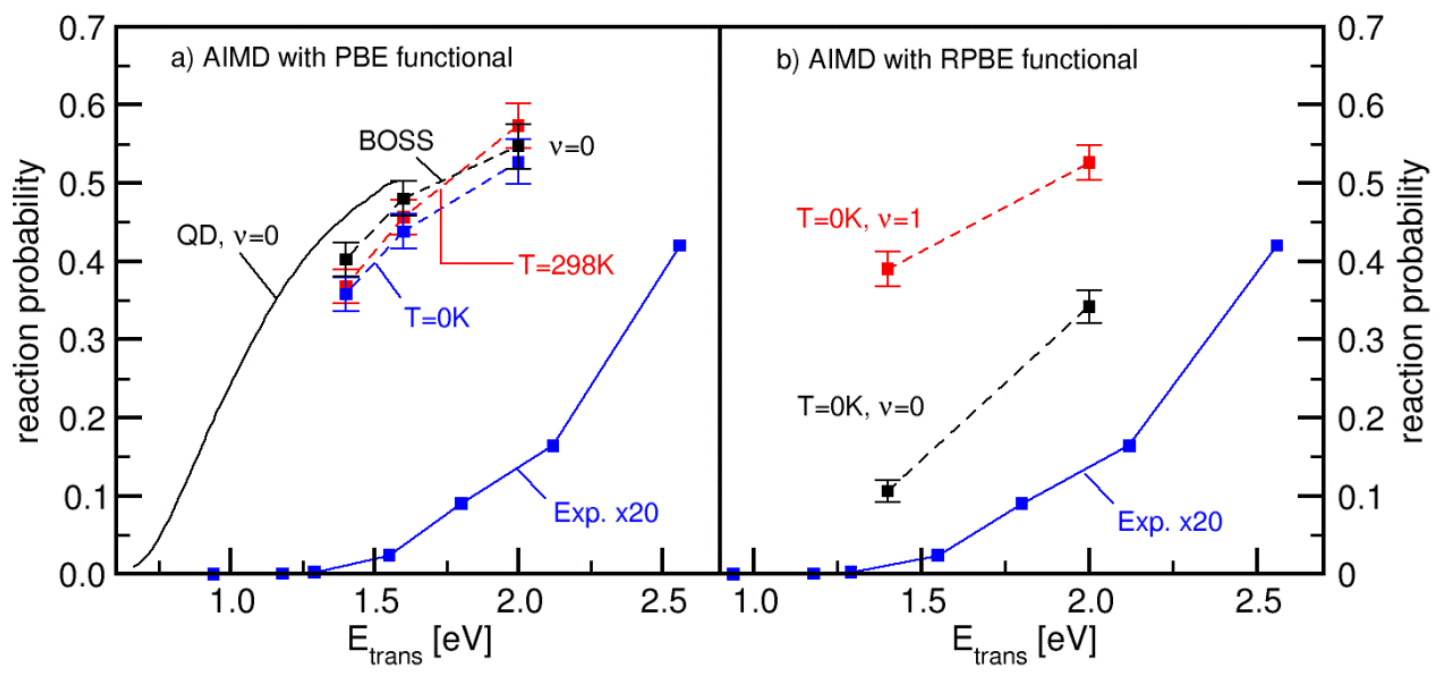

Figure 8: Reaction probabilities as functions of the incident translational energy of $\mathrm{HCl}$ scattering from $\mathrm{Au}(111)$. Shown are results taken from experiments ${ }^{29}$ (blue solid line, multiplied by a factor of 20) and computed by AIMD simulations in a) using the PBE functional and b) the RPBE functional. In panel a) we investigate the role of surface atom motion for the reactivity of $\mathrm{HCl}$ in $v=0$ by considering i) a rigid surface within the BOSS model (black dashed line), ii) the surface at $T_{S}=0 \mathrm{~K}$ and iii) the surface at $T_{S}=298 \mathrm{~K}$. We also plot previous $6 \mathrm{D}$ quantum results ${ }^{41}$ based $^{2}$ on the PW91 functional (black solid line). Panel b) shows the influence of vibrational pre-excitation of $\mathrm{HCl}$ in the gas phase on its reactivity on $\mathrm{Au}(111)$. 
Very recently another AIMD study of the reactive scattering of $\mathrm{DCl}$ on $\mathrm{Au}(111)$ has been published $^{104}$ using RPBE, but a different computational setup was employed. The energy transferred from DCl to surface atom motion was found to amount to $33-43 \%$ of the incidence translational energy which ranged from $1.25-2.5 \mathrm{eV}$. This is somewhat larger than our findings for $\mathrm{HCl}$. Experimentally the transferred energy corresponds to about $55 \%$ of the collision energy ${ }^{39}$ ranging from 0.28 to $1.27 \mathrm{eV}$, slightly larger than the Baule limit predicts. Although the amount of energy dissipated is still rather large, the dissipative phonon channel seems not to act efficiently enough on the dynamics along the reaction coordinate to have a significant influence on the reactivity of $\mathrm{HCl}$. Here a change of about 0.03-0.05 has been achieved in the reaction probability. From Ref. ${ }^{104}$, which also used the RPBE functional, a change of 0.07 can be extracted at $E_{\text {trans }}=2.5 \mathrm{eV}$ (the energy transferred to the surface was about $1100 \mathrm{meV}$ ). We will return to the point of the low efficiency with which the dissipative phonon channel inhibits reaction when we discuss electronically non-adiabatic effects.

In Figure 8a, we also plot experimental reaction probabilities ${ }^{29}$. As can be seen, the PBEAIMD reaction probabilities are much too large compared to experiment. The same is true for the previous six-dimensional quantum dynamics (QD) results based on the similar PW91 functional $^{41}$. Due to the lower (not converged) barriers, the QD results are shifted by about $115 \mathrm{meV}$ to lower values of $E_{\text {trans }}$ compared to our AIMD simulations, but the shapes of the $S$-curves are in good agreement (see also Figure 9a).

Figure $8 \mathrm{~b}$ shows AIMD results obtained with the RPBE functional. As can be seen for $v=0$ simulations, the reaction probability is considerably lower for the RPBE than for the PBE 
calculations. At $E_{\text {trans }}=1.4 \mathrm{eV}$, the reaction probability is now only 0.11 instead of 0.36 (PBE), a clear result of the increased barrier heights $\left(E^{\ddagger}=1050 \mathrm{meV}\right.$ for RPBE).

Table 3: Comparison of AIMD and some MDEF results. Listed are the reaction probabilities $S$ (with standard deviation) and the different total energy losses $E_{\text {loss }}$ obtained for the different incidence translational energies (monochromatic distribution), models and DFT functionals. The AIMD energy losses mediated by phonon-excitation are resolved for different scattering angles $\theta=0-5^{\circ}$ (specular peak along the surface normal direction), and $\Theta=17.5$ $22.5^{\circ}$. The total loss of energy taken from MDEF simulations is given for scattered and adsorbed trajectories separately. In all cases, we assume a monochromatic beam distribution. Reaction probabilities obtained with MD simulations appear in brackets.

\begin{tabular}{|c|c|c|c|}
\hline$E_{\text {trans }}[e V]$ & 1.4 & 1.6 & 2.0 \\
\hline \multicolumn{4}{|c|}{ AIMD PBE $(v=0)$} \\
\hline$S$, rigid surface & $0.402 \pm 0.022$ & $0.480 \pm 0.022$ & $0.547 \pm 0.029$ \\
\hline$S, T_{S}=0 K$ & $0.358 \pm 0.021$ & $0.438 \pm 0.022$ & $0.527 \pm 0.029$ \\
\hline$S, T_{S}=298 K$ & $0.368 \pm 0.022$ & $0.456 \pm 0.022$ & $0.573 \pm 0.029$ \\
\hline$E_{\text {loss }}[\mathrm{meV}], \mathrm{T}_{S}=0 \mathrm{~K}$, total & $456 \pm 2 / 32.5 \%$ & $539 \pm 3 / 33.7 \%$ & $701 \pm 4 / 35.0 \%$ \\
\hline$E_{\text {loss }}[\mathrm{meV}], T_{S}=298 \mathrm{~K}$, total & $426 \pm 8 / 30.4 \%$ & $519 \pm 9 / 32.4 \%$ & $679 \pm 15 / 33.9 \%$ \\
\hline$E_{\text {loss }}[\mathrm{meV}], \mathrm{T}_{S}=0 \mathrm{~K}, \boldsymbol{\theta}=0-5^{\circ}$ & $458 \pm 5$ & $542 \pm 8$ & $681 \pm 7$ \\
\hline$E_{\text {loss }}[\mathrm{meV}], \mathrm{T}_{S}=0 \mathrm{~K}, \theta=17.5-22.5^{\circ}$ & $502 \pm 8$ & $578 \pm 9$ & $711 \pm 8$ \\
\hline$E_{\text {loss }}^{B a u l e}[$ meV] & 738 & 843 & 1054 \\
\hline \multicolumn{4}{|c|}{ MDEF (MD) PBE ( $v=0, T_{e l}=0 K$, rigid surface) } \\
\hline$S(v=0)$ & $0.397(0.412)$ & $0.472(0.485)$ & $0.554(0.559)$ \\
\hline$E_{\text {loss }}[m e V]$, scat. traj. & 28.6 & 27.0 & 23.8 \\
\hline$E_{\text {loss }}[m e V]$, ads. traj. & 57.5 & 60.9 & 65.5 \\
\hline \multicolumn{4}{|c|}{ AIMD RPBE at $T_{S}=0 \mathrm{~K}$} \\
\hline$S(v=0)$ & $0.106 \pm 0.014$ & - & $0.342 \pm 0.021$ \\
\hline$S(v=1)$ & $0.390 \pm 0.022$ & - & $0.526 \pm 0.022$ \\
\hline$E_{\text {loss }}[m e V],(v=0)$ & $396 \pm 1 / 28 \%$ & - & $636 \pm 2 / 32 \%$ \\
\hline$E_{\text {loss }}[\mathrm{meV}], \mathrm{T}_{S}=0 \mathrm{~K}, \boldsymbol{\theta}=0-5^{\circ}$ & $234 \pm 2$ & - & $612 \pm 6$ \\
\hline$E_{\text {loss }}[\mathrm{meV}], T_{S}=0 \mathrm{~K}, \theta=17.5-22.5^{\circ}$ & $427 \pm 9$ & - & $674 \pm 12$ \\
\hline$E_{\text {loss }}[\mathrm{meV}],(\boldsymbol{v}=1)$ & $409 \pm 3$ & - & $644 \pm 4$ \\
\hline
\end{tabular}

Reaction probabilities presented for DCl in Ref. ${ }^{104}$ are considerably lower than our values for $\mathrm{HCl}$ although a lower barrier of $910 \mathrm{meV}$ was calculated therein. At $E_{\text {trans }}=1.25 \mathrm{eV}$, for example, an $S$-value of $1 \times 10^{-4}$ was given, and at $E_{\text {trans }}=2.0 \mathrm{eV}$ the $S$-value was about 0.16. For the latter case, we find $S=0.34$. This discrepancy might reflect the larger mass of $\mathrm{DCl}$, but we also note that the very recent AIMD simulations were purely classical, that is, 
zero-point energy was not explicitly accounted for, which should certainly lead to the computed reaction probability being diminished. Instead a classical Maxwell-Boltzmann distribution for $T=300 \mathrm{~K}$ was assumed for the atomic velocities associated to the rotational and vibrational motion of $\mathrm{DCl}$ in the gas phase. As will be shown below, however, the computed reaction probability critically depends on the initial conditions.

Table 4: Vibrational efficacies $\chi_{v}$ for $\mathrm{HCl}$ on $\mathrm{Au}(111)$. Listed values are extracted from previous 6D quantum results presented in Ref. ${ }^{41}$ (based on PW91) and from this work either obtained from AIMD results or from MD results, the latter being based on PBE. Given are the reaction probabilities $S(v=0)$ and $S(v=1)$ calculated at the incidence translational energies $E_{\text {trans }}^{\nu=0}$ and $E_{\text {trans }}^{\nu=1}$

\begin{tabular}{|l|c|c|c|c|c|}
\hline & $\boldsymbol{S}(\boldsymbol{v}=\mathbf{0})$ & $\boldsymbol{E}_{\text {trans }}^{\boldsymbol{v}=\boldsymbol{e} \boldsymbol{V}]}$ & $\boldsymbol{S}(\boldsymbol{v}=\mathbf{1})$ & $\boldsymbol{E}_{\text {trans }}^{\boldsymbol{v}=\mathbf{1} \boldsymbol{V}]}$ & $\chi_{\boldsymbol{v}}$ \\
\hline AIMD, RPBE & 0.342 & 2.000 & 0.390 & 1.400 & $>1.7$ \\
\hline MD, PBE & 0.559 & 2.120 & 0.553 & 1.180 & 2.7 \\
\hline 6D QD, Ref. $^{\mathbf{4 1}}$ & 0.111 & 0.836 & 0.111 & 0.385 & 1.3 \\
\hline 6D QD, Ref. $^{\mathbf{4 1}}$ & 0.342 & 1.144 & 0.342 & 0.625 & 1.5 \\
\hline 6D QD, Ref. $^{\mathbf{4 1}}$ & 0.503 & 1.587 & 0.503 & 0.890 & 2.0 \\
\hline
\end{tabular}

According to the Polanyi rules, the vibrational pre-excitation of $\mathrm{HCl}$ would promote the bond cleavage on $\mathrm{Au}(111)$ as the barrier to dissociation is late. This has indeed been confirmed by both theory ${ }^{41}$ and experiments ${ }^{29}$. The latter ones even suggest that the vibrational efficacy is much larger than predicted by the recent QD simulations. In Figure 8b we also plot AIMD results for $v=1$ excited $\mathrm{HCl}$. At $E_{\text {trans }}=1.4 \mathrm{eV}$, the reactivity increases from $0.11(v=0)$ to $0.39(v=1)$, which is still larger than the reaction probability of 0.34 we obtain at $E_{\text {trans }}=2.0 \mathrm{eV}$ for $v=0$. Taking into account that the transition energy from the vibrational ground to the first excited vibrational state for $\mathrm{HCl}$ is about $350 \mathrm{meV}$, we then can conclude that the added vibrational energy promotes the reaction at least 1.7 times as efficiently as a corresponding amount of translational energy added. From the previous quantum simulations a value of about 1.5 can be extracted for a similar reaction probability, but at a lower collision 
energy. For a further reduced collision energy, the 6D QD calculations yield a vibrational efficacy of 1.3 - see Table 4 for more details.

Table 5: Listed is the excess charge in $e^{-}$transferred from the metal surface to the dissociating molecule at the TS for various gas-surface systems and DFT functionals. The values are calculated as the difference between the charge of the molecule in the gas phase and the charge of the molecule at the TS. The charges are obtained from a Bader charge analysis of the electron densities computed with DFT. Values given in brackets are obtained from single point calculations using a $(3 \times 3)$ supercell and a $9 \times 9 \times 1$ k-point sampling, but for geometries taken from $(2 \times 2)$ NEB/DIMER calculations.

\begin{tabular}{|c|c|c|c|c|}
\hline & PBE & RPBE & SRP48 $^{\mathbf{8 4}}$ & SRP32-vdW $^{\mathbf{1 4}}$ \\
\hline HCl on $\mathrm{Au}(111)$ & $0.292(0.320)$ & $0.320(0.348)$ & - & - \\
\hline $\mathrm{H}_{2}$ on $\mathrm{Cu}(\mathbf{1 1 1})$ & - & - & 0.229 & - \\
\hline $\mathrm{CH}_{4}$ on $\mathrm{Ni}(111)$ & - & - & - & 0.241 \\
\hline
\end{tabular}

Although RPBE yields larger barriers than PBE, the computed reaction probabilities are still strikingly different from the experimental values. Theoretical work on $\mathrm{O}_{2}+\mathrm{Al}(111)^{25}$ suggests that GGA functionals might fail at accurately determining the reaction barrier for $\mathrm{HCl}+\mathrm{Au}(111)$, if there is a considerable charge transfer (CT) from the metal surface to $\mathrm{HCl}$ at the $\mathrm{TS}^{28}$. With the help of a Bader charge analysis we tested whether a large CT occurs. We calculated the difference between the charge associated with $\mathrm{HCl}$ in the gas phase and at the TS discussed in Sec. 3.1. From Table 5 where the corresponding values are listed, we can see that the electronic charge on the molecule indeed increases at the TS compared to the gas phase, by about $0.3 e^{-}$for both functionals. The accumulated charge is not sensitive to changes of the supercell size used in the calculations (see Table 5). For the $\mathrm{O}_{2}$ on $\mathrm{Al}(111)$ system a slightly larger value of about $0.5 e^{-}$was found ${ }^{25}$. At first sight, this might indicate the presence of a CT complex for $\mathrm{HCl}$ on $\mathrm{Au}(111)$. At this point it is interesting to look at the charges accumulated on the dissociating molecule at the TS in other gas-surface systems, for which DFT has been shown to work accurately. As examples, the corresponding values for $\mathrm{H}_{2}$ on $\mathrm{Cu}(111)^{84}$ and for $\mathrm{CH}_{4}$ on $\mathrm{Ni}(111)^{14}$ are also listed in Table 5. For both we obtain 
somewhat smaller electronic charges on the dissociating molecules of about $0.23-0.24$. More research is needed to establish whether an extra charge of $0.3 e^{-}$on the dissociating molecule at the TS is enough to be indicative of problems arising from a DFT treatment, as the differences with the two systems for which DFT works well is rather small.

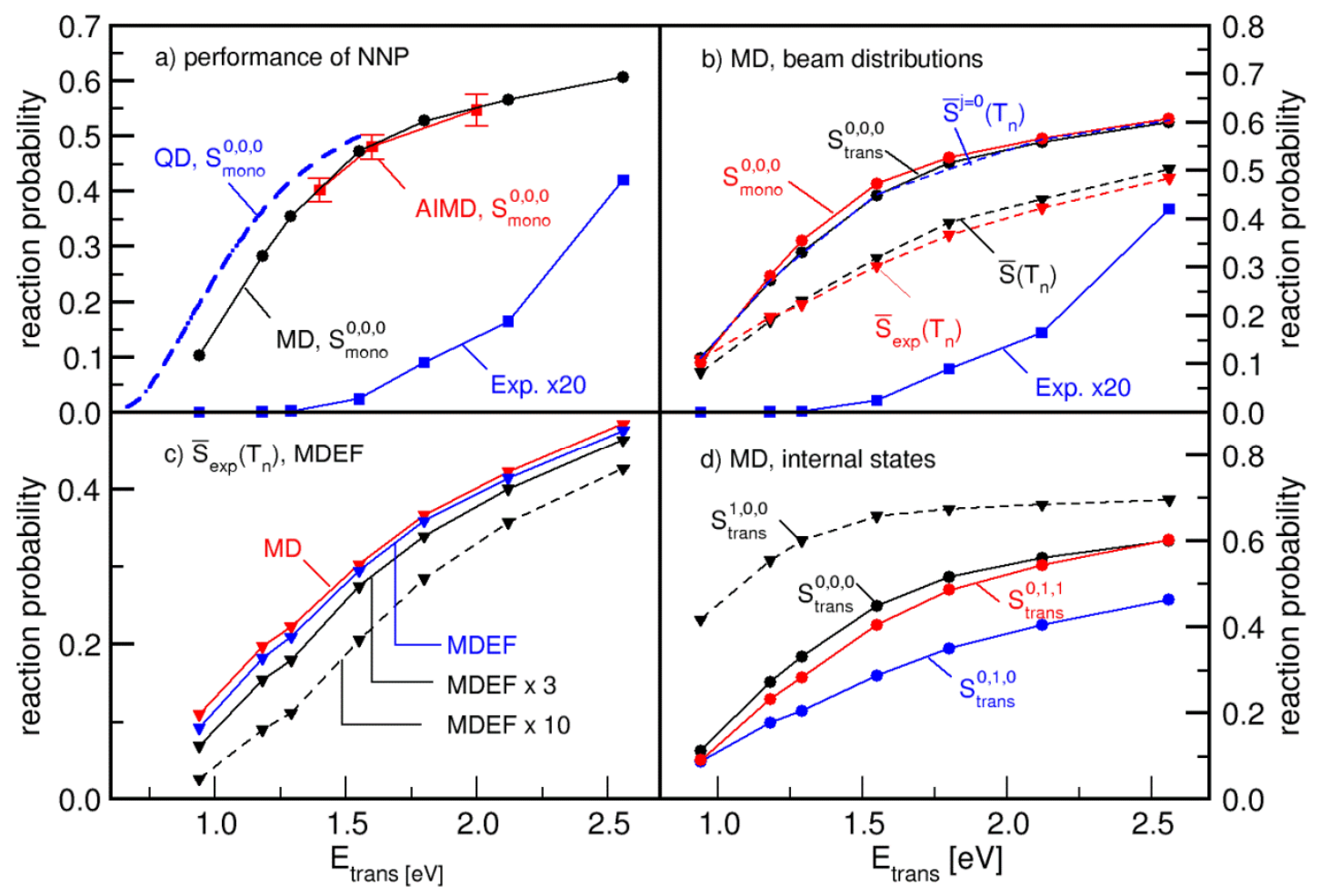

Figure 9: Reaction probabilities $S$ as functions of the (average) translational energy obtained for MD and MDEF simulations performed on the 6D NNP (PBE functional). In panel a) previous $6 \mathrm{D}$ quantum simulations ${ }^{41}$ (QD, blue dashed line), experimental results ${ }^{29}$ multiplied by a factor 20 (blue solid line) are compared with results computed with PBE-AIMD (red) and MD (black). Panel b) shows $S$ obtained from MD simulations assuming different molecular beam distributions. The notations of the different beams are as follows: i) translationally monochromatic beam for a particular rovibrational state $\left(s_{m o n o}^{v, j, m_{j}}\right)$, ii) translational distribution according to experiments but with molecules in a particular rovibrational state $\left(S_{\text {trans }}^{v, j, m_{j}}\right)$, iii) translational beam distribution like ii) and internal degrees of freedom $\left(\bar{S}\left(T_{n}\right)\right)$ populated according to the nozzle temperature $T_{n}$, iv) translation like ii) and thermally populated vibrational states only, that is, $j=0\left(\bar{S}^{j=0}\left(T_{n}\right)\right)$, and finally, v) a beam distribution according to the experiments at which $T_{v i b} \neq T_{\text {rot }}\left(\bar{S}_{\text {exp }}\left(T_{n}\right)\right)$, see text. In panel c) we present MD (red) and MDEF results with friction coefficients multiplied by different factors $(\times 1=$ blue; $\times 3=$ black solid; $\times 10=$ black dashed $)$ and $T_{e l}=170 \mathrm{~K}$. Panel d $)$ shows $S$ taken from MD simulations for $\mathrm{HCl}$ initially in different rovibrational states. 


\subsubsection{MD and MDEF results}

In this section, we discuss the influence of different rovibrational state populations of $\mathrm{HCl}$, in the incidence molecular beam, and study the importance of the dissipative ehp excitation channel for the reactive scattering of $\mathrm{HCl}$ on $\mathrm{Au}(111)$. Before discussing details, we point to Figure 9a in which we compare PBE-AIMD and 6D MD results using the neural network potential (NNP), both for the BOSS model, a monochromatic beam (single incident energy), and $\mathrm{HCl}$ in $(v=0, j=0)$. The good agreement between the MD and the AIMD results confirms that the NNP accurately describes the molecule-surface interaction, suggesting that the static surface approximation MD should be as accurate as AIMD, while offering greater statistical accuracy.

We first consider reaction probabilities for different beam distributions and internal state populations (see Figure $9 \mathrm{~b}$ ). It turns out that the inclusion of a translational energy distribution according to the experiments has only a small influence on the computed reaction probability, when comparing with monochromatic beam results, where in both cases $\mathrm{HCl}$ is in the rovibrational ground state. Allowing additionally for a vibrational state population (but with $j=0$ ) according to the nozzle temperature $T_{n}$ yields very similar results, although vibrational excitation is found to promote the reaction (see Figure 9d). This is due to the high computed reactivity of $v=0 \mathrm{HCl}$, and the large quantum of vibration (350 $\mathrm{meV}$ for the transition from the ground to the first excited vibrational state ${ }^{69}$ ) leading to low populations of excited vibrational states even at large values of $T_{n}$. We find, for example, at $E_{\text {trans }}=2.56 \mathrm{eV}$ $\left(T_{n}=1060 \mathrm{~K}\right)$ an increase of the vibrational energy of only $8 \mathrm{meV}$ (population of the $v=1$ state is about 0.022 ) compared to molecules in $v=0$, and, thus, no additional contribution of vibrational energy to the reaction is achieved in our calculations. If, however, the full ro- 
vibrational state population according to the nozzle temperature (denoted by $\bar{S}\left(T_{n}\right)$ ) is incorporated in the beam simulation, then we find that the probability for bond cleavage is reduced. At $E_{\text {trans }}=0.95 \mathrm{eV}$ this effect is small, but at $E_{\text {trans }}=2.56 \mathrm{eV}$, we observe that $\bar{S}\left(T_{n}\right)$ is about 0.1 lower than for beams with molecules in $v=0$ and $j=0$. For simulations in which we assume the vibrational and the rotational temperatures to be different according to state-resolved measurements (see Sec. 2.3.3), a similar reduction in the reactivity is observed.

The observed dependence of the reactivity on the increased population of excited rovibrational states (actually of excited rotational states) is so large that it deserves a more detailed discussion. In Figure 9d, we show results for molecules in different rotational states (translational energy distribution is according to the experiments). The molecules in the helicopter rotational states $\left(j=1, m_{j}= \pm 1\right)$ exhibit similar reactivity as molecules in the rotational ground state. Molecules in a cartwheel-like rotational state $\left(j=1, m_{j}=0\right)$ are, however, less reactive. This means that the overall effect of adding rotational energy to the molecule is to decrease its reactivity. The difference between the reactivity of the $j=1$ cartwheel state and that of the rotational ground state is similar in magnitude to the difference found between the reactivity of the $(v=0, j=0)$ state and that of rotationally excited nozzle beams (see Figure 9b). The difference between the reactivity of the $j=1$ helicopter and cartwheel state is in qualitative agreement with previous observations made by Zhang and coworkers $^{41}$, who investigated the dependence of the $\mathrm{HCl}$ dissociation on the different initial rotational states associated with $j=5$. The observed $m_{j}$ dependence suggest that rotational alignment effects are at work in the dynamics. As discussed in Sec. 3.1, most of the barriers lying between 750 and $1000 \mathrm{meV}$ are most easily traversed if the molecule impacts with $\theta \in[110,135]^{\circ}$, that is, if the molecule is initially aligned more parallel (helicopter motion) 
to the surface than perpendicular (cartwheel motion). The clearly distinct reactivity of molecules in the different rotational states further suggests that the shape of the PES does not enforce rotational steering effects for the high incidence energies considered.

While exciting the rotational states seems to act counterproductively on the bond cleavage, strong enhancement is achieved by exciting molecules to the $v=1$ state prior to collision, as shown in Figure 9d. To quantify this, an excitation to $v=1$ at $E_{\text {trans }}=1.18 \mathrm{eV}$ leads to a similar reaction probability as a translational excitation to $E_{\text {trans }}=2.12 \mathrm{eV}(v=0)$ (see MD results in Table 4). The corresponding vibrational efficacy $\chi_{v}$ is 2.5 and larger than the values found in 6D QD simulations and our RPBE-AIMD calculations presented, but note that the vibrational efficacy depends on the value of the reaction probability at which it is evaluated. Our calculations suggest that the effect of both the initial rotational and the initial vibrational state should be taken into account in the simulation of molecular beam experiments on sticking of $\mathrm{HCl}$ to $\mathrm{Au}(111)$. However, the associated effects are not strong enough to account for the large discrepancies observed between the theory and the experiments.

The AIMD simulations presented in the previous section suggest that energy transfer to surface phonons, while not unimportant, does not have a dramatic impact on the reactivity. With the help of MDEF simulations employing the LDFA-IAA approximation, we now investigate the importance of ehp excitation for the dynamics while assuming $T_{e l}=170 \mathrm{~K}$, according to the experimental surface temperature. The results are summarized in Figure 9c. Comparing MDEF and MD reaction probabilities shows that the dissociation process is not much affected by non-adiabatic effects at the LDFA level of theory. On average the MDEF differ by about 0.01-0.02 from the MD results (see Table 3 ). This could be a consequence of the underlying LDFA approach, which may yield too low values of the friction coefficients 
because changes in the electronic structure of the molecule with bond stretching near the TS are not accounted for ${ }^{106}$.

Very recently additional experimental evidence for non-adiabatic effects near the TS was found for $\mathrm{HCl}$ scattering from $\mathrm{Au}(111)^{107}$. However, the new measurements do not provide direct evidence of electronically non-adiabatic effects for the surface temperature used in the sticking experiments, at which vibrational excitation is predominantly adiabatic. In order to test how a fictive model yielding larger values of the friction coefficients might perform, we evaluated the dynamics with increased friction coefficients by scaling the LDFA values with a factor 3 and 10 , as done earlier for $\mathrm{H}_{2}$ on $\mathrm{Ru}(0001)^{20}$. Our results clearly suggest that the reactivity may be inhibited if ehp excitations become increasingly possible: the larger the nonadiabatic coupling is, the lower the reactivity of $\mathrm{HCl}$ is observed to be.

The presence of electronic friction hardly changes the shape of the MDEF-curves as a function of the translational energy. Instead we find them to be shifted to each other along the energy axis. This is different to previous observations for the early barrier of the $\mathrm{H}_{2}+$ $\mathrm{Ru}(0001)$ system $^{20}$ for which MDEF and MD simulations showed a similar amount of bond cleavage at very low and very high incidence energies. The difference in behavior could be related to how friction acts in an early and in a late barrier system. In an early barrier system, the presence of friction leads to a velocity dependent barrier height. In a late barrier system, electronic friction might also inhibit the energy transfer from the motion perpendicular to the reaction path (initially, the molecular vibration) to motion along the reaction path (initially, the motion towards the surface). This might reduce the capability of the molecule to overcome the reaction barrier. 
We now recall that phonon excitation may lead to a large amount of energy being dissipated to the surface (about $30 \%$ of the initial incidence translational energy), but this had only a small influence on the reaction probability (the change being 0.03-0.05). Comparing the $S$ values obtained from MD and MDEF simulations listed in Table 3, the inclusion of friction according to the LDFA might seem to be unimportant on both counts, but the associated loss of energy makes for less than $2 \%$ of $E_{\text {trans }}$, while the reactivity is still reduced by $0.01-0.02$. In this sense, the electronic dissipative channel interferes much more efficiently with the nuclear dynamics to reduce the reactivity than the phonon channel. The latter mechanism is most likely linked with the motion of the $\mathrm{Cl}$ atom, due to its large mass, while the former mechanism acts on the $\mathrm{H}$ atom, due to its ability to penetrate deeper into the electron density. It is the hydrogen motion that allows for the dissociation of $\mathrm{HCl}$. Electronic friction therefore acts along the reaction coordinate, and if it was as effective for energy dissipation as phonon excitation, as observed to be the case of the scaled LDFA model, then it would most likely be more effective at inhibiting the reaction. For example, at $E_{\text {trans }}=1.29 \mathrm{eV}$, increasing the friction by a factor of 10 reduces the reaction probability from 0.21 to 0.11 with normal LDFA friction, while the loss of energy for scattered trajectories increases by about $120 \mathrm{meV}$, from $27 \mathrm{meV}$ to $150 \mathrm{meV}$. These values need to be seen in a context where there are also energy losses due to the excitation of the surface atom motion upon impact of $\mathrm{HCl}$.

We also mention that electronically non-adiabatic effects provide a possible explanation for the experimentally observed translational energy losses for $\mathrm{HCl}$ scattering from $\mathrm{Au}(111)$ (55\% of the incidence translational energy) being larger than energy losses predicted in our AIMD simulations (about 30\%), and in the Baule limit (50\% of the incidence translational energy). When adding, for example, the amount of energy loss of $456 \mathrm{meV}$ taken from PBEAIMD simulations at an incidence energy of $1.4 \mathrm{eV}$ to the energy loss of $27 \mathrm{meV}$ and 150 
$m e V$, respectively, taken from (scaled) MDEF simulations at $1.29 \mathrm{eV}$, the total energy loss becomes 483 and $606 \mathrm{meV}$, respectively, depending on the scaling factor of the friction coefficient. This is close to the experimental value of about $700 \mathrm{meV}$ at $E_{\text {trans }}=1.27 \mathrm{eV}^{39}$ obtained for the experimental specular scattering angle (the angle made between the surface normal and the scattering direction is $\Theta=3^{\circ}$ in the experiments). We also looked in more detail at the angular dependence of the energy losses for scattered trajectories. The $\mathrm{HCl}$ molecules are found to scatter not too far from the specular direction $\left(\Theta_{\mathrm{f}}\right.$ is peaked at about 5$10^{\circ}$ along the surface normal direction). Total energy losses obtained for different final scattering angle $\Theta_{\mathrm{f}}$ values and incidence translational energies are listed in Table 3 for PBEAIMD and RPBE-AIMD simulations at $T_{S}=0 K$. The energy losses for specular scattering agree reasonably well with the averaged energy losses, while somewhat larger losses are found for increased scattering angles. If we assume that the somewhat larger amount of energy loss found experimentally is due to the presence of non-adiabaticity, this would suggest that the LDFA friction coefficients are too small or that a treatment using electronic friction is insufficient. This either suggests that the LDFA model underestimates the importance of electronic friction, or that attractive interactions are present due to the presence of van der Waals London dispersion. This can lead to deep adsorption wells in the entrance channel. As a consequence, $\mathrm{HCl}$ would gain additional kinetic energy which then can be dissipated to the surface via both the electronic and the phonon channel.

We finally need to consider the possibility that non-adiabaticity is incorrectly taken into account in our current MDEF simulations due to the physics we employ in the friction limit. In electron scattering experiments on $\mathrm{HCl}$ in the gas phase, vibrational excitation is achieved by shape resonances at low collision energies $(0.8 \mathrm{eV})^{108,109}$. The associated vibrational cross sections are found to be large ${ }^{108}$. This might suggest that a non-adiabatic electron hopping 
approach coupled to ehp excitation such as proposed in Ref. ${ }^{5}$ might be a better way of incorporating non-adiabatic effects than the LDFA-IAA approach for the $\mathrm{HCl}+\mathrm{Au}(111)$ system.

\subsection{Discussion: disagreement between theory and experiment}

In this Section we will consider how the theory may be improved to bring it in better agreement with experiment, but before we do that we first discuss the accuracy of the experimental measurements, as this topic has not yet received much attention in the preceding part of our paper.

Two important sources of error in the experiments concern the calibration of the coverage of $\mathrm{Au}(111)$ by $\mathrm{Cl}$ resulting from dissociative adsorption of $\mathrm{HCl}$, and the calibration of the flux of the impinging $\mathrm{HCl}$. Starting with the first point, we think that an error may have been made in the calibration of the coverage. For this, Auger spectroscopy was used ${ }^{29}$. More specifically, the Auger peak-to-peak height ratio $P_{r}=I_{C l} / I_{A u}$ for $\mathrm{Cl}$ at $181 \mathrm{eV}$ and $\mathrm{Au}$ at $239 \mathrm{eV}$ was used for this purpose. The unnormalized coverage of the surface by chlorine was computed from measured peak ratios and known sensitivity factors $\mathrm{Q}_{\mathrm{Au}}$ and $\mathrm{Q}_{\mathrm{Cl}}$ for the radiation used, as described in the supporting information to Ref. ${ }^{29}$. However, to obtain the normalized coverage required for evaluating sticking coefficients of $\mathrm{HCl}$, a reference is needed. This reference was based on measurements of relative Auger intensities, which were presented in Figure 3 of a paper by Spencer and Lambert ${ }^{110}$, and are reproduced in Figure 10a. In the procedure used, Shirhatti et al. used the highest value of the relative intensity (of about 8) as the value corresponding to saturation coverage of $\mathrm{Au}(111)$ by chlorine, and assumed this coverage to correspond to 1 monolayer ${ }^{29}$.

A general problem with this reference is that Spencer and Lambert clearly stated in their paper that their Auger intensity ratios should not be used for calibration purposes, because they 
believed that at the highest $\mathrm{Cl}_{2}$ exposures used in their experiments on the $\mathrm{Cl}_{2} / \mathrm{Au}(111)$ system multiple layers of a surface compound of $\mathrm{Au}$ and $\mathrm{Cl}$ should be present on the surface ${ }^{110}$. A recommendation that seems to be implicit in their work is that if their data is nevertheless to be used for calibration purposes, this is best done on the assumption of an initial sticking coefficient of $\mathrm{Cl}_{2}$ on $\mathrm{Au}(111)$ of 1 , based on analogous findings for similar systems (Ref. ${ }^{110}$ and references therein). We will now proceed on this basis. It is convenient to initially think in terms of an unnormalized surface concentration of $\mathrm{Cl}_{2}$ on the surface, which may be expressed as ${ }^{29}$

$\left[C l_{2}\right]_{u n}=K \frac{P_{r} Q_{A u}}{P_{r} Q_{A u}+Q_{C l}}$

where the proportionality constant $K=10^{19} \mathrm{~m}^{-2}$ is merely used here to ensure that the unnormalized $\mathrm{Cl}_{2}$ concentration appears in units that are convenient to the subsequent analysis. A plot of $\left[\mathrm{Cl}_{2}\right]_{\text {un }}$ appears in Figure $10 \mathrm{~b}$. We define the normalized $\mathrm{Cl}_{2}$ concentration analogously as

$\left[C l_{2}\right]=\frac{K}{N} \frac{P_{r} Q_{A u}}{P_{r} Q_{A u}+Q_{C l}}$

where $N$ is the dimensionless normalization factor that needs to be determined. This can be done by realizing that

$\left[C l_{2}\right]\left(x^{\prime}\right)=\int_{x=0}^{x \prime} S(x) d x$

where $x$ is $\mathrm{Cl}_{2}$ exposure in $10^{19}$ molecules $\mathrm{m}^{-2}$ (and the reason for introducing the proportionality constant $K$ is now becoming apparent), and $S(x)$ is the exposure dependent sticking coefficient, which may also be defined as $S(x)=d\left[\mathrm{Cl}_{2}\right] / d x$. The use of this definition of the sticking coefficient and Eq.( 30 ) is appropriate under conditions where any $\mathrm{Cl}_{2}$ molecule adsorbed to the surface remains on the surface in some form of chlorine for the duration of the experiment, which assumption would seem to be justified in view of the large adsorption energy of $\mathrm{Cl}_{2}$ on $\mathrm{Au}(111)^{111}$. Requiring that $S(x=0)=1$ identifies the normalization 
coefficient $\mathrm{N}$ as the initial slope of $\left[\mathrm{Cl}_{2}\right]_{\text {un }}$ vs. $x$ in Figure 10b, for which a linear least squares fit to the data computed from Eq.( 28 ) yields $N=0.09245$. Using this value of $\mathrm{N}$ yields $\left[\mathrm{Cl}_{2}\right]$ vs. $x$ in Figure 10c (left y-axis) and, trivially, $[\mathrm{Cl}]$ vs. $x$ (right y-axis). As can be seen, a reference value of 8 for $P_{r}$ corresponds to $[\mathrm{Cl}] \approx 4.5 \times 10^{19}$ atoms $\mathrm{m}^{-2}$. Using that 1 monolayer $(\mathrm{ML})=1.39 \times 10^{19}$ atoms $\mathrm{m}^{-2}$ (as obtainable from the nearest neighbour $\mathrm{Au}-\mathrm{Au}$ distance in the unreconstructed $(1 \times 1)-\mathrm{Au}(111)$ surface of $\left.2.88 \AA^{111}\right)$, this corresponds to 3.2 ML, suggesting that the values reported for the sticking coefficients in Ref. ${ }^{29}$ should be multiplied by a factor 3.2. This problem may be exacerbated if the $\mathrm{HCl}$ sticking probabilities were determined from coverages exceeding 0.2 ML, as our factor of 3.2 and the raw data in Figure 1 of Ref. ${ }^{29}$ would seem to suggest for some of the conditions used in the experiments.

The correctness of the above analysis relies on the initial sticking coefficient of $\mathrm{Cl}_{2}$ on $\mathrm{Au}(111)$ at room temperature being equal to 1, the Auger intensity ratio not being dependent on the form chlorine is in at the surface (atomic initially, $\mathrm{AuCl}_{3}$ at higher exposures ${ }^{110,112}$ ), and the instrument sensitivies ${ }^{29}$ for the $\mathrm{Cl}$ and $\mathrm{Au}$ Auger peaks being identical, where the latter assumption has also been used by Shirhatti et al. ${ }^{29}$. Kastanas and Koel reported finding that the sticking coefficient slightly decreases with substrate temperature (the gas temperature in their experiments being room temperature ${ }^{111}$. Using their reported negative apparent activation energy for $\mathrm{Cl}_{2}$ dissociative chemisorption to compute the slope of the curve of the sticking coefficient vs. $T_{S}$ and assuming a sticking coefficient of 1 for the lowest $T_{S}$ addressed $(120 \mathrm{~K})^{111}$, we find a sticking coefficient of 0.6 at room temperature. This would suggest that the coverage used to describe saturation in the $\mathrm{HCl}+\mathrm{Au}(111)$ experiments would correspond to only 1.9 ML, and that the sticking coefficients would be underestimated by a factor of about 2. In any case, a better way of referencing an Auger signal to some standard for coverage is suggested by the work of Gao et al. ${ }^{112}$, who determined that $\mathrm{Cl}$ on $\mathrm{Au}(111)$ should exhibit a specific LEED pattern at 1/3 ML coverage, which could then be linked to a reference Auger intensity ratio. Obviously, future theoretical work aimed at establishing the level of theory needed to reproduce and interpret experiments on sticking of $\mathrm{HCl}$ to $\mathrm{Au}(111)$ will benefit from the availability of experimental results that are as accurate as possible. 


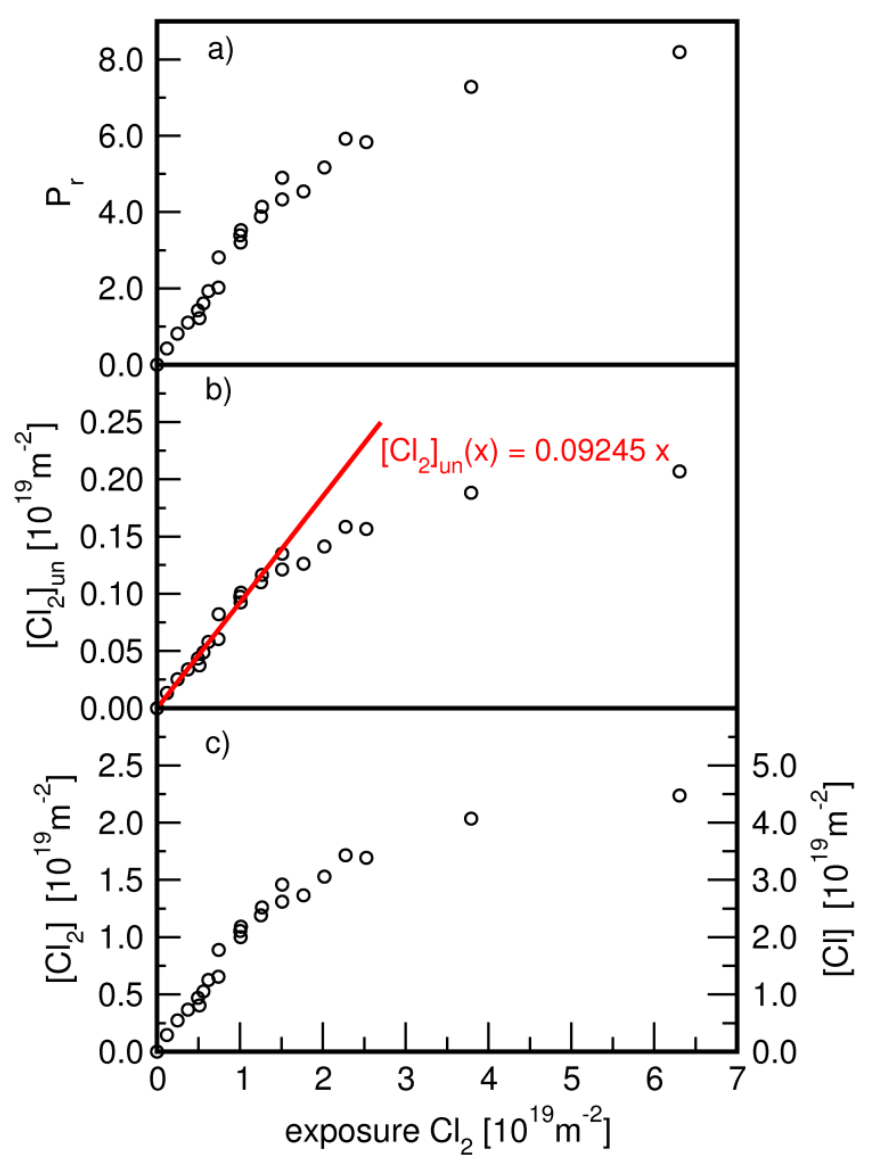

Figure 10: Plotted as functions of the $\mathrm{Cl}_{2}$ exposure are the relative Auger peak-to-peak ratios $\boldsymbol{P}_{r}$ for $\mathrm{Cl}(181 \mathrm{eV})$ and $\mathrm{Au}(239 \mathrm{eV})$ taken from Ref. ${ }^{110}$ (a), the unnormalized concentration $\left[\mathrm{Cl}_{2}\right]_{\text {un }}$ adsorbed on the gold surface together with the linear fit (red line) to obtain the normalization factor $N(\mathrm{~b})$, and the normalized concentration $\left[\mathrm{Cl}_{2}\right]($ left $\mathrm{y}$ axis) and $[C l]$ (right $y$ axis) (c). For further information see the text.

Proceeding to the second point (flux calibration), we note that in the experiments measured signals had to be divided by a factor ranging from 6-10 to account for the hydrodynamic enrichment of $\mathrm{HCl}$ in the beam. To estimate how much more $\mathrm{HCl}$ should be present in the molecular beam hitting the surface relative to the original $\mathrm{HCl} / \mathrm{H}_{2}$ mixture, experiments were done using $\mathrm{Ar}$ in $\mathrm{H}_{2}$, arguing that a similar hydrodynamic enrichment factor would be expected for $\mathrm{HCl}$ and $\mathrm{Ar}^{29}$. While this may be correct, we argue that the size of the correction factors (in range 6-10) is such that the verification of this similarity might be called for in future experiments.

In their own error analysis, the experimentalists noted possible systematic errors of $50 \%$ due to absolute pressure calibration and of $30 \%$ in estimates of the pumping speed. They 
estimated that these errors limited the accuracy in their determined sticking probabilities to $\pm 60 \%$. It is not clear whether this was based on assuming the errors to propagate as statistical errors. If the systematic errors work in the same direction, their combined effect could be another factor 2. Elsewhere in their paper, the experimentalists also mentioned that their sticking probabilities should be multiplied by a factor of up to 1.5 if the assumption they made that recombinative desorption of $\mathrm{HCl}$ is unimportant at the surface temperature used $(170 \mathrm{~K})$ should be incorrect. In the worst-case scenario the combined systematic errors leads to a systematic error of another factor of 3 . Combined with the factor 3.2 due to the possibly incorrect external standard for coverage of $\mathrm{Au}$ by $\mathrm{Cl}$ and a possible uncertainty in the dynamic enrichment of $\mathrm{HCl}$ in the beam, the maximum sticking probability could be considerably larger than the reported measured value of 0.02 . We advocate that future experiments on $\mathrm{HCl}$ $+\mathrm{Au}(111)$ eliminate as much of the uncertainties described above, to allow the best possible comparison to future calculations.

Before moving to the analysis of how the theory can be improved, we make one more remark regarding the experiments. The present analysis of the extent to which vibrational preexcitation should enhance the dissociative chemisorption of $\mathrm{HCl}$ on $\mathrm{Au}(111)$ also depends on the validity of the approximation that the reaction only depends on the component of the translational energy normal to the surface (normal energy scaling, NES). The vibrational efficacy of $\mathrm{HCl}$ on $\mathrm{Au}(111)$ could be put on a more solid basis if experiments were to be carried out in which a substantial proportion of the incident molecules would be vibrationally pre-excited in the incident beam by a laser, analogous to what has been done in experiments on methane reacting with metal surfaces ${ }^{113,114}$. 


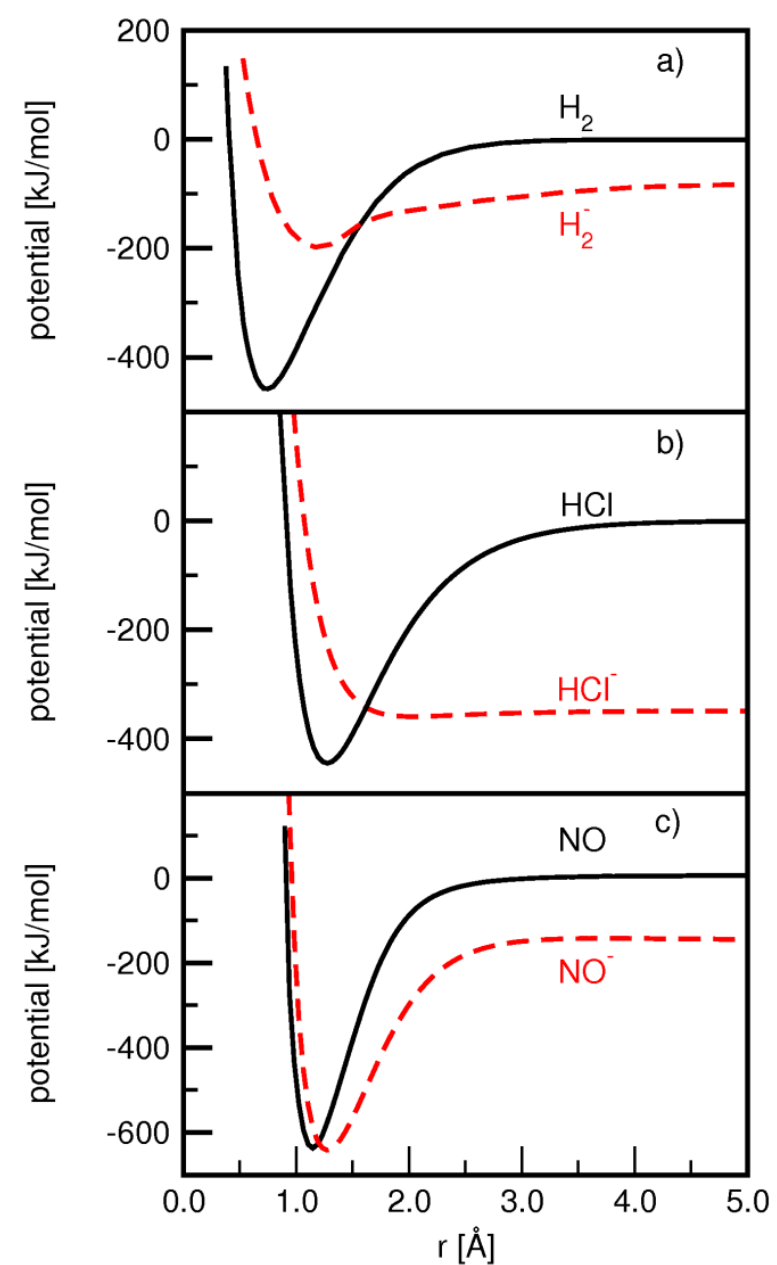

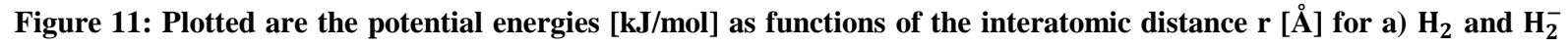
taken from Ref. ${ }^{115}$, for b) $\mathrm{HCl}$ and $\mathrm{HCl}^{-}$according to Ref. ${ }^{116}$, and for c) NO and $\mathrm{NO}^{-}$taken from Ref. ${ }^{4}$.

We now turn to the theory, where we will be more brief as much of what we will mention now has already come up earlier. It might be worthwhile to experiment with different functionals, which yield higher and later barriers. An example of a functional that could be tested is a functional combining PBE and RPBE exchange with a correlation functional ${ }^{117}$ yielding an approximately correct description of the attractive van der Waals interaction, as recently done successfully for methane reacting on $\mathrm{Ni}(111)^{14}$. This might also fix the problem of the presently incorrect description of the $\mathrm{HCl}-\mathrm{Au}(111)$ physisorption minimum, the orientationally averaged well depth of which should be much higher (at $220 \mathrm{meV}^{35}$ ) than here found with PBE and RPBE. Introducing the van der Waals interaction might tend to steer the incident molecule more to geometries where the $\mathrm{Cl}$ atom points down, which could help to 
reduce the reactivity. As already hinted at, it might also be a good idea to explore the use of $a b$ initio based electronic structure methods ${ }^{25,118,119}$ to see whether their use would yield higher, and possibly later, barriers for the $\mathrm{HCl}+\mathrm{Au}(111)$ system, the transition state of which is characterized by at least a partial charge transfer.

It may also be fruitful to explore different methods for dealing with possible electronically non-adiabatic effects. The first thing that comes to mind is to try methods that are still based on the idea of electronic friction, but employ orbital-based, or tensorial friction coefficients ${ }^{106}$, for instance using a recent efficient implementation to evaluate them ${ }^{92}$. Alternatively, the independent electron surface hopping (IESH) method of Tully and co-workers ${ }^{5}$, which is designed to deal with a situation where a charge transfer state is coupled to a continuum of electron-hole pair excited states, could be tested. It is not yet clear whether the use of this method should be necessary: in terms of where the ground state gas phase molecule and molecular anion potential curves cross relative to the gas phase molecular minimum, $\mathrm{HCl}^{116}$ is intermediate to $\mathrm{NO}$ and $\mathrm{H}_{2}$ (see Figure 11 and Ref. ${ }^{120}$ and references therein). In any case a non-adiabatic model would need to reconcile the observations that non-adiabatic effects apparently strongly hinder the reaction of $v=0 \mathrm{HCl}$ (as suggested by the present and earlier comparisons between adiabatic theory and experiment, and likely involving a deceleration of $\mathrm{HCl}$ bond stretching) while, on the other hand, the reaction of vibrationally excited $\mathrm{HCl}$ is enormously enhanced over reaction of $v=0 \mathrm{HCl}$. Perhaps a proper description of the potential transfer of an electron to the molecule would show the reaction to be hindered by reorientation effects if the transfer would take place to the $\mathrm{Cl}$ atom. On the other hand the immediate expected effect would be bond lengthening ${ }^{116}$, which would be expected to promote reaction rather than hinder it. Closing off the discussion of the treatment of nonadiabatic effects, we note that the use of classical dynamics might be problematic regardless of whether a friction or a surface hopping approach is used. The accurate treatment of electronically non-adiabatic vibrationally inelastic effects may well require the use of quantum mechanics, as employed for instance in the reduced density matrix calculations of NO scattering from $\mathrm{Au}(111)^{121}$. In future, the present and other theoretical methods to deal 
with electronic non-adiabaticity could also be tested in comparisons with experiments measuring energy losses ${ }^{35,39}$ and vibrationally inelastic scattering at high surface temperatures ${ }^{107}$ in scattering of $\mathrm{HCl}$ from $\mathrm{Au}(111)$.

Finally, theory could move closer to experiment if some of the effects noted here could accumulate. For instance, in ab initio molecular dynamics with electronic friction (AIMDEF) calculations $^{90,122}$, energy transfer to phonons and electron-hole pairs could act in concert to hinder reaction, whether using LDFA or tensorial friction. These AIMDEF calculations could also model the effect of the rotational state distribution in the incident beam, which we predict should further diminish the reactivity. It is quite possible that AIMDEF calculations properly modeling friction and incorporating the effects of rotations would predict reaction probabilities in much better agreement with the reported experimental sticking probabilities. As stressed already above, such a comparison would also benefit from the availability of measured sticking probabilities with less uncertainties in them, for instance with better calibration of the sticking probabilities based on more accurately evaluated coverages of $\mathrm{Au}$ by product $\mathrm{Cl}$ atoms, and of incident $\mathrm{HCl}$ flux.

\section{Conclusions}

In this work, we performed quasiclassical dynamics simulations for the reactive scattering of $\mathrm{HCl}$ on $\mathrm{Au}(111)$ at normal incidence to test the influence of several methodological aspects. With the help of AIMD simulations, we investigated the importance of the GGA functional and the effects of allowing surface motion and of surface temperature on the computed reaction probabilities. We also generated a 6D PES in the framework of the rigid surface approximation by fitting a large amount of DFT points obtained from PBE calculations with the neural network approach. The availability of a PES also allowed us to study the effect of molecular beams with different translational and rovibrational state population distributions, 
and the effect of electron hole pair excitation on the reactivity of $\mathrm{HCl}$ on gold. From our analysis and the discussion in Section 3.3 the following conclusions can be drawn:

i) Barriers calculated with PBE and PW91 on the one hand are lower than the ones calculated with RPBE on the other hand. As a result, the reaction probabilities are lower for the latter functional. The $\mathrm{TS}$ of $\mathrm{HCl}$ on $\mathrm{Au}(111)$ is late from the viewpoint of adsorption, explaining the largely enhanced reactivity for molecules vibrationally excited to the $v=1$ state compared to molecules in the vibrational ground state. Additionally, the orientation of the TS in $\theta$ suggests that the dissociation is preferred for molecules aligned parallel to the surface rather than perpendicular. There is modest evidence found of somewhat greater transfer of charge from the metal to the dissociating $\mathrm{HCl}$ molecule at the $\mathrm{TS}$ than found for systems for which DFT works well. More research is needed to establish whether the amount of charge transferred is large enough for GGA-DFT to break down, which then might cause underprediction of the barrier height.

ii) The AIMD results for reaction show no strong dependence on the inclusion of surface atom motion or on the surface temperature. The amount of energy dissipating from the molecules to the surface atoms is large and makes for about $30 \%$ of the incidence translational energy of the molecule, but the change in reactivity was small compared to the rigid surface calculations. This suggests a small coupling of the phonon dissipation channel with the reaction coordinate.

iii) MD simulations assuming different molecular beam distributions suggest that the internal rotational state population is important for the reactivity of $\mathrm{HCl}$ on $\mathrm{Au}(111)$. This is a result of the $\theta$-dependence of the barrier making the 
dissociation less likely if the molecule is aligned perpendicular to the surface. Rotational states that are associated with the cartwheel motion of the molecule exhibit less reactivity. Due to the late barriers, bond cleavage is, on the other hand, promoted if excited vibrational states are significantly populated. At the experimental nozzle temperatures, however, the population of vibrationally excited states is still rather low, due to the large vibrational quantum of $\mathrm{HCl}$.

iv) From the non-adiabatic MDEF simulations, we can conclude that ehp excitation has no considerable effect on the dynamics at the LDFA level of theory. The electronic-nuclear coupling strength is too small (or the impact time too short) to allow for a large amount of energy dissipation from the molecule to the surface electrons. Non-adiabatic effects become significant, however, if the friction coefficients are scaled by a factor of 10 . It is not yet clear whether such large couplings, or even larger couplings, would be obtained with a method providing tensorial friction that accounts for the molecular structure.

v) The AIMD calculations underestimate the amount of energy transferred to the surface in scattering compared to experimental observations, and the difference is not accounted for by energy losses observed in MDEF simulations.

vi) We suggest that the reported measured sticking coefficients are underestimated by a factor of about 2-3 due to a possibly erroneous calibration of coverage of Au by $\mathrm{Cl}$.

Altogether, we conclude that, in spite of the possibility that the reported experimental sticking probabilities could be underestimated by a factor of $2-3$, none of the presented simulations yield reaction probabilities comparable to the experiments, and the origin of the discrepancy 
with the experiments is still unclear. The DFT functional and the rovibrational state population in the molecular beam experiment show the largest influence. If we assume the optimistic case that both would work additively then we can reduce the reaction probability computed at $E_{\text {trans }}=2.0 \mathrm{eV}$ from $S$ is about $0.5\left(\mathrm{PBE}, T_{S}=0 \mathrm{~K}\right.$, monochromatic beam and $v=j=0$ ) to $S$ is about 0.2 . This is still far from the experimental value of $S=0.0082$ at $E_{\text {trans }}=2.12 \mathrm{eV}$. Future work on this system might require the use of other DFT functionals, or of a quantum chemical method similar to that used in Ref. ${ }^{25}$, which yield larger barriers. Also the possibility of "anti"-steering effects should be considered as an explanation for the large discrepancies, that is, forces resulting from the PES in the entrance channel which may rotate the molecule away from orientations favorable for reaction, as might be obtained with functionals accounting for vdW-DF correlations ${ }^{117}$, accounting for attractive London dispersion interactions. Finally, one should also consider the possibility that there are errors in the measured reaction probabilities that are larger than estimated.

\section{Acknowledgements}

We gratefully acknowledge Jörg Meyer for providing a computer code for the neural network fit and for fruitful discussions. We further thank Jan Geweke and Pranav Ravindra Shirhatti for providing a rotational temperature fit based on state-resolved data for $\mathrm{HCl}$ in the beam and for many discussions. We are grateful to Daniel Auerbach and Bruce Koel for useful discussions. Francesco Nattino is acknowledged for providing the Bader charge analysis of $\mathrm{H}_{2}$ on $\mathrm{Cu}(111)$ and $\mathrm{CH}_{4}$ on $\mathrm{Ni}(111)$. This work was supported financially by the Nederlandse Organisatie voor Wetenschappelijk Onderzoek (NWO-CW) through a TOP grant and by the European Research Council through an ERC-2013 advanced grant (Nr. 338580), and with computer time granted by NWO-EW. M. del Cueto acknowledges the FPI program of the 
MICINN, co-financed by the ESF. C. Díaz acknowledges the MICINN project CTQ2013-

50150-EXP and the Ramón y Cajal program.

\section{References}

1. Anderson, P. W. Infrared Catastrophe in Fermi Gases with Local Scattering Potentials. Phys. Rev. Lett. 1967, 18, 1049-1051.

2. Hasselbrink, E., Capturing the Complexities of Molecule-Surface Interactions. Science 2009, 326, 809-810.

3. Gergen, B.; Nienhaus, H.; Weinberg, W. H.; McFarland, E. W. Chemically Induced Electronic Excitations at Metal Surfaces. Science 2001, 294, 2521-2523.

4. Huang, Y. H.; Rettner, C. T.; Auerbach, D. J.; Wodtke, A. M. Vibrational Promotion of Electron Transfer. Science 2000, 290, 111-114.

5. Shenvi, N.; Roy, S.; Tully, J. C. Dynamical Steering and Electronic Excitation in NO Scattering from a Gold Surface. Science 2009, 326, 829-832.

6. White, J. D.; Chen, J.; Matsiev, D.; Auerbach, D. J.; Wodtke, A. M. Conversion of Large-Amplitude Vibration to Electron Excitation at a Metal Surface. Nature 2005, 433, 503505 .

7. Nahler, N. H.; White, J. D.; Larue, J.; Auerbach, D. J.; Wodtke, A. M. Inverse Velocity Dependence of Vibrationally Promoted Electron Emission from a Metal Surface. Science 2008, 321, 1191-1194.

8. Bünermann, O.; Jiang, H. Y.; Dorenkamp, Y.; Kandratsenka, A.; Janke, S. M.; Auerbach, D. J.; Wodtke, A. M. Electron-Hole Pair Excitation Determines the Mechanism of Hydrogen Atom Adsorption. Science 2015, 350, 1346-1349.

9. Kroes, G. J.; Pavanello, M.; Blanco-Rey, M.; Alducin, M.; Auerbach, D. J. Ab Initio Molecular Dynamics Calculations on Scattering of Hyperthermal $\mathrm{H}$ Atoms from $\mathrm{Cu}(111)$ and $\mathrm{Au}(111)$. J. Chem. Phys. 2014, 141, 054705.

10. Janke, S. M.; Auerbach, D. J.; Wodtke, A. M.; Kandratsenka, A. An Accurate FullDimensional Potential Energy Surface for H-Au(111): Importance of Nonadiabatic Electronic Excitation in Energy Transfer and Adsorption. J. Chem. Phys. 2015, 143, 124708.

11. Díaz, C.; Pijper, E.; Olsen, R. A.; Busnengo, H. F.; Auerbach, D. J.; Kroes, G. J. Chemically Accurate Simulation of a Prototypical Surface Reaction: $\mathrm{H}_{2}$ Dissociation on $\mathrm{Cu}(111)$. Science 2009, 326, 832-834.

12. Sementa, L.; Wijzenbroek, M.; van Kolck, B. J.; Somers, M. F.; Al-Halabi, A.; Busnengo, H. F.; Olsen, R. A.; Kroes, G. J.; Rutkowski, M.; Thewes, C., et al. Reactive Scattering of $\mathrm{H}_{2}$ from $\mathrm{Cu}(100)$ : Comparison of Dynamics Calculations Based on the Specific Reaction Parameter Approach to Density Functional Theory with Experiment. J. Chem. Phys. 2013, 138, 044708.

13. Wijzenbroek, M.; Kroes, G. J. The Effect of the Exchange-Correlation Functional on $\mathrm{H}_{2}$ Dissociation on $\mathrm{Ru}(0001)$. J. Chem. Phys. 2014, 140, 084702.

14. Nattino, F.; Migliorini, D.; Kroes, G. J.; Dombrowski, E.; High, E. A.; Killelea, D. R.; Utz, A. L. Chemically Accurate Simulation of a Polyatomic Molecule-Metal Surface Reaction. J. Phys. Chem. Lett. 2016, 7, 2402-2406.

15. Hundt, P. M.; Jiang, B.; van Reijzen, M. E.; Guo, H.; Beck, R. D. Vibrationally Promoted Dissociation of Water on Ni(111). Science 2014, 344, 504-507.

16. Nattino, F.; Ueta, H.; Chadwick, H.; van Reijzen, M. E.; Beck, R. D.; Jackson, B.; van Hemert, M. C.; Kroes, G. J. Ab Initio Molecular Dynamics Calculations Versus Quantum- 
State-Resolved Experiments on $\mathrm{CHD}_{3}+\mathrm{Pt}(111)$ : New Insights into a Prototypical GasSurface Reaction. J. Phys. Chem. Lett. 2014, 5, 1294-1299.

17. Nave, S.; Tiwari, A. K.; Jackson, B. Dissociative Chemisorption of Methane on Ni and Pt Surfaces: Mode Specific Chemistry and the Effects of Lattice Motion. J. Phys. Chem. A 2014, 118, 9615-9631.

18. Luntz, A. C.; Persson, M. How Adiabatic Is Activated Adsorption/Associative Desorption. J. Chem. Phys. 2005, 123, 074704.

19. Juaristi, J. I.; Alducin, M.; Díez Muiño, R.; Busnengo, H. F.; Salin, A. Role of Electron-Hole Pair Excitations in the Dissociative Adsorption of Diatomic Molecules on Metal Surfaces. Phys. Rev. Lett. 2008, 100, 116102.

20. Füchsel, G.; Schimka, S.; Saalfrank, P. On the Role of Electronic Friction for Dissociative Adsorption and Scattering of Hydrogen Molecules at a Ru(0001) Surface. J. Phys. Chem. A 2013, 117, 8761-8769.

21. Muzas, A. S.; Juaristi, J. I.; Alducin, M.; Diéz Muiño, R.; Kroes, G. J.; Díaz, C. Vibrational Deexcitation and Rotational Excitation of $\mathrm{H}_{2}$ and $\mathrm{D}_{2}$ Scattered from $\mathrm{Cu}(111)$ : Adiabatic Versus Non-Adiabatic Dynamics. J. Chem. Phys. 2012, 137, 064707.

22. Jiang, B.; Alducin, M.; Guo, H. Electron-Hole Pair Effects in Polyatomic Dissociative Chemisorption: Water on Ni(111). J. Phys. Chem. Lett. 2016, 7, 327-331.

23. Behler, J.; Delley, B.; Lorenz, S.; Reuter, K.; Scheffler, M. Dissociation of $\mathrm{O}_{2}$ at Al(111): The Role of Spin Selection Rules. Phys. Rev. Lett. 2005, 94, 036104.

24. Behler, J.; Reuter, K.; Scheffler, M. Nonadiabatic Effects in the Dissociation of Oxygen Molecules at the Al(111) Surface. Phys. Rev. B 2008, 77, 115421.

25. Libisch, F.; Huang, C.; Liao, P. L.; Pavone, M.; Carter, E. A. Origin of the Energy Barrier to Chemical Reactions of $\mathrm{O}_{2}$ on $\mathrm{Al}(111)$ : Evidence for Charge Transfer, Not Spin Selection. Phys. Rev. Lett. 2012, 109, 198303.

26. Honkala, K.; Hellman, A.; Remediakis, I. N.; Logadottir, A.; Carlsson, A.; Dahl, S.; Cristensen, C. H.; Nørskov, J. K. Ammonia Synthesis from First-Principles Calculations. Science 2005, 307, 555-558.

27. Jones, G.; Jacobsen, J. G.; Shim, S. S.; Kleis, J.; Andersson, M. P.; Rossmeisl, J.; Abild-Pedersen, F.; Bligaard, T.; Helveg, S.; Hinneman, B., et al. First Principles Calculations and Experimental Insight into Methane Steam Reforming over Transition Metal Catalysts. $J$. Catal. 2008, 259, 147-160.

28. Wodtke, A. M. Electronically Non-Adiabatic Influences in Surface Chemistry and Dynamics. Chem. Soc. Rev. 2016.

29. Shirhatti, P. R.; Geweke, J.; Steinsieck, C.; Bartels, C.; Rahinov, I.; Auerbach, D. J.; Wodtke, A. M. Activated Dissociation of $\mathrm{HCl}$ on $\mathrm{Au}(111)$. J. Phys. Chem. Lett. 2016, 7, 1346-1350.

30. Rettner, C. T. Reaction of an H-Atom Beam with $\mathrm{Cl} / \mathrm{Au}$ (111): Dynamics of Concurrent Eley-Rideal and Langmuir-Hinshelwood Mechanisms. J. Chem. Phys. 1994, 101, 1529-1546.

31. Rettner, C. T.; Auerbach, D. J. Distinguishing the Direct and Indirect Products of a Gas-Surface Reaction. Science 1994, 263, 365-367.

32. Jackson, B.; Persson, M. Quantum Mechanical Study of H(G)+Cl-Au(111): EleyRideal Mechanism. J. Chem. Phys. 1994, 100, 7687-7695.

33. Lemoine, D.; Quattrucci, J. G.; Jackson, B. Efficient Eley-Rideal Reactions of H Atoms with Single Cl Adsorbates on $\mathrm{Au}(111)$. Phys. Rev. Lett. 2002, 89, 268302.

34. Quattrucci, J. G.; Jackson, B.; Lemoine, D. Eley-Rideal Reactions of H Atoms with Cl Adsorbed on $\mathrm{Au}(111)$ : Quantum and Quasiclassical Studies. J. Chem. Phys. 2003, 118, 2357.

35. Lykke, K. R.; Kay, B. D. Rotationally Inelastic Gas-Surface Scattering: HCl from $\mathrm{Au}(111)$. J. Chem. Phys. 1990, 92, 2614-2623. 
36. Roscioli, J. R.; Bell, D. J.; Nelson, D. J.; Nesbitt, D. J. State-Resolved Velocity Map Imaging of Surface-Scattered Molecular Flux. Phys. Chem. Chem. Phys. 2012, 14, 40704080 .

37. Ran, Q.; Matsiev, D.; Auerbach, D. J.; Wodtke, A. M. Observation of a Change of Vibrational Excitation Mechanism with Surface Temperature: $\mathrm{HCl}$ Collisions with $\mathrm{Au}(111)$. Phys. Rev. Lett. 2007, 98, 237601.

38. Rahinov, I.; Cooper, R.; Yuan, C.; Yang, X. M.; Auerbach, D. J.; Wodtke, A. M. Efficient Vibrational and Translational Excitations of a Solid Metal Surface: State-to-State Time-of-Flight Measurements of $\mathrm{HCl}(\mathrm{v}=2, \mathrm{~J}=1)$ Scattering from $\mathrm{Au}(111)$. J. Chem. Phys. 2008, 129, 214708.

39. Cooper, R.; Rahinov, I.; Yuan, C.; Yang, X. M.; Auerbach, D. J.; Wodtke, A. M. Efficient Translational Excitation of a Solid Metal Surface: State-to-State Translational Energy Distributions of Vibrational Ground State $\mathrm{HCl}$ Scattering from $\mathrm{Au}(111)$. J. Vac. Sci. Technol. A 2009, 27, 907-912.

40. Ran, Q.; Matsiev, D.; Auerbach, D. J.; Wodtke, A. M. Direct Translation-toVibrational Energy Transfer of $\mathrm{HCl}$ on Gold: Measurement of Absolute Vibrational Excitation Probabilities. Nucl. Instrum. Methods Phys. Res., Sect. B 2007, 258, 1-6.

41. Liu, T. H.; Fu, B. N.; Zhang, D. H. Six-Dimensional Quantum Dynamics Study for the Dissociative Adsorption of $\mathrm{HCl}$ on $\mathrm{Au}(111)$ Surface. J. Chem. Phys. 2013, 139, 184705.

42. Liu, T. H.; Fu, B. N.; Zhang, D. H. Six-Dimensional Quantum Dynamics Study for the Dissociative Adsorption of DCl on Au(111) Surface. J. Chem. Phys. 2014, 140, 144701.

43. Liu, T. H.; Fu, B. N.; Zhang, D. H. Six-Dimensional Potential Energy Surface of the Dissociative Chemisorption of $\mathrm{HCl}$ on $\mathrm{Au}(111)$ Using Neural Networks. Sci. China: Chem 2014, 57, 147-155.

44. Perdew, J. P.; Chevary, J. A.; Vosko, S. H.; Jackson, K. A.; Pederson, M. R.; Singh, D. J.; Fiolhais, C. Atoms, Molecules, Solids, and Surfaces: Applications of the Generalized Gradient Approximation for Exchange and Correlation. Phys. Rev. B 1992, 46, 6671-6687.

45. Herzberg, G. Molecular Spectra and Molecular Structure. Volume 1 - Spectra of Diatomic Molecules; Krieger: Malabar, 1989.

46. Perdew, J. P.; Burke, K.; Ernzerhof, M. Generalized Gradient Approximation Made Simple. Phys. Rev. Lett. 1996, 77, 3865-3868.

47. Hammer, B.; Hansen, L. B.; Nørskov, J. K. Improved Adsorption Energetics within Density-Functional Theory Using Revised Perdew-Burke-Ernzerhof Functionals. Phys. Rev. B. 1999, 59, 7413-7421.

48. Tang, W.; Sanville, E.; Henkelman, G. A Grid-Based Bader Analysis Algorithm without Lattice Bias. J. Phys.: Condens. Matter 2009, 21, 084204.

49. Sanville, E.; Kenny, S. D.; Smith, R.; Henkelman, G. An Improved Grid-Based Algorithm for Bader Charge Allocation. J. Comput. Chem. 2007, 28, 899-908.

50. Henkelman, G.; Arnaldsson, A.; Jónsson, H. A Fast and Robust Algorithm for Bader Decomposition of Charge Density. Comput. Mater. Sci. 2006, 36, 254-360.

51. Yu, M.; Trinkle, D. R. Accurate and Efficient Algorithm for Bader Charge Integration. J. Chem. Phys. 2011, 134, 064111.

52. Behler, J. Neural Network Potential-Energy Surfaces in Chemistry: A Tool for LargeScale Simulations. Phys. Chem. Chem. Phys. 2011, 13, 17930-17955.

53. Lorenz, S.; Groß, A.; Scheffler, M. Representing High-Dimensional Potential-Energy Surfaces for Reactions at Surfaces by Neural Networks. Chem. Phys. Lett. 2004, 395, 210215.

54. Michelsen, H. A.; Rettner, C. T.; Auerbach, D. J.; Zare, R. N. Effect of Rotation on the Translational and Vibrational Energy Dependence of the Dissociative Adsorption of $\mathrm{D}_{2}$ on Cu(111). J. Chem. Phys. 1993, 98, 8294-8307. 
55. Rettner, C. T.; Michelsen, H. A.; Auerbach, D. J. Quantum State Specific Dynamics of the Dissociative Adsorption and Associative Desorption of $\mathrm{H}_{2}$ at a $\mathrm{Cu}(111)$ Surface. J. Chem. Phys. 1995, 102, 4625-4641.

56. Busnengo, H. F.; Salin, A.; Dong, W. Representation of the 6D Potential Energy Surface for a Diatomic Molecule near a Solid Surface. J. Chem. Phys. 2000, 112, 7641-7651.

57. Luppi, M.; Olsen, R. A.; Baerends, E. J. Six-Dimensional Potential Energy Surface for $\mathrm{H}_{2}$ at $\mathrm{Ru}(0001)$. Phys. Chem. Chem. Phys. 2006, 8, 688-696.

58. Füchsel, G.; Tremblay, J. C.; Saalfrank, P. A Six-Dimensional Potential Energy Surface for $\mathrm{Ru}(0001)(2 \times 2)$ :CO. J. Chem. Phys. 2014, 141, 094704.

59. Shen, X. J.; Lozano, A.; Dong, W.; Busnengo, H. F.; Yan, X. H. Towards Bond Selective Chemistry from First Principles: Methane on Metal Surfaces. Phys. Rev. Lett. 2014, $112,046101$.

60. Shen, X. J.; Chen, J.; Zhang, Z. J.; Shao, K. J.; Zhang, D. H. Methane Dissociation on Ni(111): A Fifteen-Dimensional Potential Energy Surface Using Neural Network Method. $J$. Chem. Phys. 2015, 143, 144701.

61. Díaz, C.; Olsen, R. A.; Auerbach, D. J.; Kroes, G. J. Six Dimensional Dynamics Study of Reactive and Non Reactive Scattering of $\mathrm{H}_{2}$ from $\mathrm{Cu}(111)$ Using a Chemically Accurate Potential Energy Surface. Phys. Chem. Chem. Phys. 2010, 12, 6499-6519.

62. Kresse, G.; Hafner, J. Ab-Initio Molecular-Dynamics for Liquid-Metals. Phys. Rev. B 1993, 47, 558-561.

63. Kresse, G.; Hafner, J. Ab Initio Molecular-Dynamics Simulation of the Liquid-MetalAmorphous-Semiconductor Transition in Germanium. Phys. Rev. B 1994, 49, 14251-14269.

64. Kresse, G.; Furthmüller, J. Efficiency of Ab-Initio Total Energy Calculations for Metals and Semiconductors Using a Plane-Wave Basis Set. Comp. Mater. Sci. 1996, 6, 15-50. 65. Kresse, G.; Furthmüller, J. Efficient Iterative Schemes for Ab Initio Total-Energy Calculations Using a Plane-Wave Basis Set. Phys. Rev. B 1996, 54, 11169-11186.

66. Blöchl, P. E. Projector Augmented-Wave Method. Phys. Rev. B 1994, 50, 1795317979.

67. Kresse, G.; Joubert, D. From Ultrasoft Pseudopotentials to the Projector AugmentedWave Method. Phys. Rev. B 1999, 59, 1758-1775.

68. Maeland, A.; Flanagan, T. B. Lattice Spacings of Gold-Palladium Alloys. Can. J. Phys. 1964, 42, 2364.

69. Irikura, K. K. Experimental Vibrational Zero-Point Energies: Diatomic Molecules. $J$. Phys. Chem. Ref. Data 2007, 36, 389.

70. Darwent, B. d. Nat. Stand. Ref. Data. Ser., 1970; Vol. 31, p. 30.

71. Burrus, G. A.; Gordy, W.; Benjamin, B.; Livingston, R. One-to-Two Millimeter Wave Spectra of TCl and TBr. Phys. Rev. 1955, 97, 1661.

72. Behler, J. Representing Potential Energy Surfaces by High-Dimensional Neural Network Potentials. J. Phys.: Condens. Matter 2014, 26, 183001.

73. Cybenko, G. Approximation by Superpositions of a Sigmoidal Function. Math. Control Signal Syst. 1989, 2, 303-314.

74. Hornik, K. Approximation Capabilities of Multilayer Feedforward Networks. Neural Netw. 1991, 4, 251-257.

75. Behler, J.; Lorenz, S.; Reuter, K. Representing Molecule-Surface Interactions with Symmetry-Adapted Neural Networks. J. Chem. Phys. 2007, 127, 014705.

76. Goikoetxea, I.; Beltrán, J.; Meyer, J.; Juaristi, J. I.; Alducin, M.; Reuter, K. NonAdiabatic Effects During the Dissociative Adsorption of $\mathrm{O}_{2}$ at $\mathrm{Ag}(111)$ ? A First-Principles Divide and Conquer Study. New J. Phys 2012, 14, 013050.

77. Jiang, B.; Guo, H. Permutation Invariant Polynomial Neural Network Approach to Fitting Potential Energy Surfaces. J. Chem. Phys. 2013, 139, 054112. 
78. Meyer, J. Ab Initio Modeling of Energy Dissipation During Chemical Reactions at Transition Metal Surfaces. Ph. D. thesis, FU Berlin, 2012.

79. Lorenz, S.; Scheffler, M.; Gross, A. Descriptions of Surface Chemical Reactions Using a Neural Network Representation of the Potential-Energy Surface. Phys. Rev. B 2006, $73,115431$.

80. Head-Gordon, M.; Tully, J. C. Molecular Dynamics with Electronic Frictions. J. Chem. Phys. 1995, 103, 10137-10145.

81. Kubo, R. The Fluctuation-Dissipation Theorem. Rep. Prog. Phys. 1966, 29, 255.

82. Press, W. H.; Teukolsky, S. A.; Vetterling, W. T.; Flannery, B. F. Numerical Recipes in Fortran, 2nd Edition; Cambridge University Press: Cambridge, 1992.

83. Ermak, D. L.; Buckholz, H. Numerical Integration of the Langevin Equation: Monte Carlo Simulation. J. Comput. Phys. 1980, 35, 169-182.

84. Nattino, F.; Díaz, C.; Jackson, B.; Kroes, G. J. Effect of Surface Motion on the Rotational Quadrupole Alignment Parameter of $\mathrm{D}_{2}$ Reacting on $\mathrm{Cu}(111)$. Phys. Rev. Lett. 2012, 108, 236104.

85. Leksina, I. E.; Novikova, S. I. Thermal Expansion of Copper, Silver, and Gold within a Wide Range of Temperatures. Sov. Phys.-Solid State 1963, 5, 798-801.

86. Colbert, D. T.; Miller, W. H. A Novel Discrete Variable Representation for Quantum Mechanical Reactive Scattering Via the S-Matrix Kohn Method. J. Chem. Phys. 1992, 96, 1982.

87. Zare, R. N. Angular Momentum: Understanding Spatial Aspects in Chemistry and Physics; Wiley: New York, 1988.

88. d'Agliano, E. G.; Kumar, P.; Schaich, W.; Suhl, H. Brownian Motion Model of the Interactions between Chemical Species and Metallic Electrons: Bootstrap Derivation and Parameter Evaluation. Phys. Rev. B 1975, 11, 2122.

89. Puska, M. J.; Nieminen, R. M. Atoms Embedded in an Electron Gas: Phase Shifts and Cross Sections. Phys. Rev. B 1983, 27, 6121.

90. Novko, D.; Blanco-Rey, M.; Alducin, M.; Juaristi, J. I. Surface Electron Density Models for Accurate Ab Initio Molecular Dynamics with Electronic Friction. Phys. Rev. B 2016, 93, 245435.

91. Rittmeyer, S. P.; Meyer, J.; Juaristi, J. I.; Reuter, K. Electronic Friction-Based Vibrational Lifetimes of Molecular Adsorbates: Beyond the Independent-Atom Approximation. Phys. Rev. Lett. 2015, 115, 046102.

92. Askerka, M.; Maurer, R. J.; Batista, V. S.; Tully, J. C. Role of Tensorial Friction in Energy Transfer at Metal Surfaces. Phys. Rev. Lett. 2016, 116, 217601.

93. Tremblay, J. C. A Unifying Model for Non-Adiabatic Coupling at Metallic Surfaces Beyond the Local Harmonic Approximation: From Vibrational Relaxation to Scanning Tunneling Microscopy. J. Chem. Phys. 2013, 138, 244106.

94. Füchsel, G.; Klamroth, T.; Monturet, S.; Saalfrank, P. Dissipative Dynamics within the Electronic Friction Approach: The Femtosecond Laser Desorption of $\mathrm{H}_{2} / \mathrm{D}_{2}$ from Ru(0001). Phys. Chem. Chem. Phys. 2011, 13, 8659-8670.

95. Füchsel, G.; Tremblay, J. C.; Klamroth, T.; Saalfrank, P.; Frischkorn, C. Concept of a Single Temperature for Highly Nonequilibrium Laser-Induced Hydrogen Desorption from a Ruthenium Surface. Phys. Rev. Lett. 2012, 109, 098303.

96. Echenique, P. M.; Nieminen, R. M.; Ritchie, R. H. Density Functional Calculation of Stopping Power of an Electron Gas for Slow Ions. Solid State Commun. 1981, 37, 779-781.

97. Nazarov, V. U.; Kim, C. S.; Takada, Y. Spin Polarization of Light Atoms in Jellium: Detailed Electronic Structures. Phys. Rev. B 2005, 72, 233205. 
98. Henkelman, G.; Uberuaga, B.; Jónsson, H. A Climbing Image Nudged Elastic Band Method for Finding Saddle Points and Minimum Energy Paths. J. Chem. Phys. 2000, 113, 9901.

99. Henkelman, G.; Jónsson, H. Improved Tangent Estimate in the Nudged Elastic Band Method for Finding Minimum Energy Paths and Saddle Points. J. Chem. Phys. 2000, 113, 9978.

100. Xiao, P.; Sheppard, D.; Rogal, J.; Henkelman, G. Solid-State Dimer Method for Calculating Solid-Solid Phase Transitions. J. Chem. Phys. 2014, 140, 174104.

101. Kästner, J.; Sherwood, P. Superlinearly Converging Dimer Method for Transition State Search. J. Chem. Phys. 2008, 128, 014106.

102. Heyden, A.; Bell, A. T.; Keil, F. J. Efficient Methods for Finding Transition States in Chemical Reactions: Comparison of Improved Dimer Method and Partitioned Rational Function Optimization Method. J. Chem. Phys. 2005, 123, 224101.

103. Henkelman, G.; Jónsson, H. A Dimer Method for Finding Saddle Points on High Dimensional Potential Surfaces Using Only First Derivatives. J. Chem. Phys. 1999, 111, 7010 .

104. Kolb, B.; Guo, H. Energy Transfer and Reaction Dynamics for DCl Scattering on Au(111): An Ab Initio Molecular Dynamics Study. J. Chem. Phys. 2016, 145, 011102.

105. Baule, B. Theoretische Behandlung der Erscheinungen in Verdünnten Gasen. Ann. Phys. (Berlin) 1914, 349, 145-176.

106. Luntz, A. C.; Makkonen, I.; Persson, M.; Holloway, S.; Bird, D. M.; Mizielinski, M. S. Comment on "Role of Electron-Hole Pair Excitations in the Dissociative Adsorption of Diatomic Molecules on Metal Surfaces". Phys. Rev. Lett. 2009, 102, 109601.

107. Geweke, J.; Shirhatti, P. R.; Rahinov, I.; Bartels, C.; Wodtke, A. M. Vibrational Energy Transfer near a Dissociative Adsorption Transition State: State-to-State Study of $\mathrm{HCl}$ Collisions at $\mathrm{Au}(111)$. J. Chem. Phys. 2016, 145, 054709.

108. Ziesel, J. P.; Nenner, I.; Schulz, G. J. Negative Ion Formation, Vibrational Excitation, and Transmission Spectroscopy in Hydrogen Halides. J. Chem. Phys. 1975, 63, 1943.

109. Shandilya, B. K.; Sarma, M.; Adhikari, S.; Mishra, M. K. Vibrational Excitation Resulting from Electron Capture in LUMO of $\mathrm{F}_{2}$ and $\mathrm{HCl}-\mathrm{a}$ Treatment Using the TimeDependent Wave Packet Approach. J. Chem. Sci. 2012, 124, 141.

110. Spencer, N. D.; Lambert, R. M. Chlorine Chemisorption and Surface Chloride Formation on $\mathrm{Au}(111)$. Surf. Sci. 1981, 107, 237.

111. Kastanas, G. N.; Koel, B. E. Interaction of $\mathrm{Cl}_{2}$ with the $\mathrm{Au}(111)$ Surface in the Temperature Range of 120 to 1000 K. Appl. Surf. Sci 1993, 64, 235.

112. Gao, W.; Baker, T. A.; Zhou, L.; Pinnaduwage, D. S.; Kaxiras, E.; Friend, C. M. Chlorine Adsorption on $\mathrm{Au}(111)$ : Chlorine Overlayer or Surface Chloride? J. Am. Chem. Soc. 2008, 130,3560 .

113. Beck, R. D.; Maroni, P.; Papageorgopoulos, D. C.; Dang, T. T.; Schmid, M. P.; Rizzo, T. R. Vibrational Mode-Specific Reaction of Methane on a Nickel Surface. Science 2003, 302, 98-100.

114. Juurlink, L. B. F.; McCabe, P. R.; Smith, R. R.; DiCologero, C. L.; Utz, A. L. Eigenstate-Resolved Studies of Gas-Surface Reactivity: $\mathrm{CH}_{4}\left(\mathrm{v}_{3}\right)$ Dissociation on $\mathrm{Ni}(100)$. Phys. Rev. Lett. 1999, 83, 868-871.

115. Čížek, M.; Horáček, J.; Domcke, W. Nuclear Dynamics of the $\mathrm{H}_{2}{ }^{-}$Collision Complex Beyond the Local Approximation: Associative Detachment and Dissociative Attachment to Rotationally and Vibrationally Excited Molecules. J. Phys. B 1998, 31, 2571-2583.

116. Fedor, J.; Winstead, C.; McKoy, V.; Č́̌žek, M.; Houfec, K.; Kolorenč, P.; Horáček, J. Electron Scattering in HCl: An Improved Nonlocal Resonance Model. Phys. Rev. A 2010, 81, 042702. 
117. Dion, M.; Rydberg, H.; Schröder, E.; Langreth, D. C.; Lundqvist, B. I. Van Der Waals Density Functional for General Geometries. Phys. Rev. Lett. 2004, 92, 246401.

118. Filippi, C.; Healy, S. B.; Kratzer, P.; Pehlke, E.; Scheffler, M. Quantum Monte Carlo Calculations of $\mathrm{H}_{2}$ Dissociation on $\mathrm{Si}(100)$. Phys. Rev. Lett. 2002, 89, 166102.

119. Doblhoff-Dier, K.; Meyer, J.; Hoggan, P. E.; Kroes, G. J.; Wagner, L. K. Diffusion Monte Carlo for Accurate Dissociation Energies of 3d Transition Metal Containing Molecules. J. Chem. Theory Comput. 2016, 12, 2583.

120. Nieto, P.; Pijper, E.; Barredo, D.; Laurent, G.; Olsen, R. A.; Baerends, E. J.; Kroes, G. J.; Farías, D. Reactive and Non-Reactive Scattering of $\mathrm{H}_{2}$ from a Metal Surface: The Electronically Adiabatic Picture. Science 2006, 312, 86-89.

121. Monturet, S.; Saalfrank, P. Role of Electronic Friction During the Scattering of Vibrationally Excited Nitric Oxide Molecules from Au(111). Phys. Rev. B 2010, 82, 075404.

122. Blanco-Rey, M.; Juaristi, J. I.; Díez Muiño, R.; Busnengo, H. F.; Kroes, G. J.; Alducin, M. Electronic Friction Dominates Hydrogen Hot-Atom Relaxation on Pd(100). Phys. Rev. Lett. 2014, 112, 103203. 